\title{
Airway administration of corticosteroids for prevention of bronchopulmonary dysplasia in premature infants: a meta-analysis with trial sequential analysis
}

\author{
Zhi-Qun Zhang ${ }^{1,2}$, Ying Zhong ${ }^{1}$, Xian-Mei Huang ${ }^{2}$ and Li-Zhong Du ${ }^{1 *}$
}

\begin{abstract}
Background: Uncertainly prevails with regard to the use of inhalation or instillation steroids to prevent bronchopulmonary dysplasia in preterm infants. The meta-analysis with sequential analysis was designed to evaluate the efficacy and safety of airway administration (inhalation or instillation) of corticosteroids for preventing bronchopulmonary dysplasia (BPD) in premature infants.

Methods: We searched MEDLINE, EMBASE, CINAHL, and Cochrane CENTRAL from their inceptions to February 2017. All published randomized controlled trials (RCTs) evaluating the effect of airway administration of corticosteroids (AACs) vs placebo or systemic corticosteroid in prematurity were included. All meta-analyses were performed using Review Manager 5.3.

Results: Twenty five RCTs retrieved ( $n=3249$ ) were eligible for further analysis. Meta-analysis and trial sequential analysis corrected the $95 \%$ confidence intervals estimated a lower risk of the primary outcome of BPD (relative risk 0.71, adjusted $95 \%$ confidence interval $0.57-0.87$ ) and death or BPD (relative risk 0.81, adjusted 95\% confidence interval $0.71-0.97$ ) in AACs group than placebo and it is equivalent for preventing BPD than systemic corticosteroids. Moreover, AACs fail to increasing risk of death compared with placebo (relative risk 0.90, adjusted $95 \%$ confidence interval $0.40-2.03$ ) or systemic corticosteroids (relative risk 0.81, 95\% confidence interval 0.62-1.06).

Conclusions: Our findings suggests that AACs (especially instillation of budesonide using surfactant as a vehicle) are an effective and safe option for preventing BPD in preterm infants. Furthermore, the appropriate dose and duration, inhalation or instillation with surfactant as a vehicle and the long-term safety of airway administration of corticosteroids needs to be assessed in large trials.
\end{abstract}

Keywords: Inhaled corticosteroids, Bronchopulmonary dysplasia, Preterm infants, Meta-analysis, Neurodevelopmental outcomes

\section{Background}

Bronchopulmonary dysplasia (BPD), defined as oxygen dependence at 36 weeks of postmenstrual age (PMA), is a severe complications of extremely premature infants. The reported morbidity of BPD ranges from $24 \%$ in preterm infants born at 28 weeks to $79 \%$ in preterm infants born at

\footnotetext{
* Correspondence: dulizhong@zju.edu.cn

'Department of Neonatology, the Children's Hospital, Zhejiang University School of Medicine, No. 3333 Bingsheng Road, Hangzhou City, Zhejiang 310002, China

Full list of author information is available at the end of the article
}

23 weeks [1].The survival infants of BPD are at high risk for long-term injury to both lung and brain [2-4]. Pulmonary inflammation plays a central, modulating role in the pathogenesis of BPD [5-7]. It has been confirmed that corticosteroids have strong anti-inflammatory effects. Randomized controlled trials (RCTs) have shown that systemic administration of corticosteroids reduces the incidence of BPD but aggravate short-term and long-term adverse effects [8-11]. Theoretically, airway administration (inhalation or instillation) of corticosteroids, on the other hand, demonstrate high pulmonary deposition, low 
systemic bioavailability, and rapid systemic clearance, thereby reducing the adverse effects $[12,13]$.

A range of reviews address the use of inhaled corticosteroids for preventing BPD $[14,15]$. Onland et al. [14] have attempted the late use ( $\geq 7$ days)) of inhaled corticosteroids to reduce $\mathrm{BPD}$ incidence in preterm infants and concluded that such treatment do not reduce the outcomes of BPD and death or BPD. Similarly, Shah et al. [15] also concluded that early ( $<2$ weeks) administration of inhaled corticosteroids was ineffective for reducing the incidence of BPD in ventilated very low birth weight premature infants. However, some overlap in ages at administration of the inhaled corticosteroid existed in the included studies in their meta-analysis. In recent, a large RCT confirmed that inhalation of budesonide reduces both BPD and persistent ductus arteriosus (PDA) [16]. However, there was a trend toward increased mortality. Subsequently, a metaanalysis including the RCT by Bassler D et al. [17] showed that inhaled corticosteroids reduced risk for BPD and had no effect on death. Their meta-analysis included several therapeutic studies and failed to evaluate the intratracheal instillation of steroids $[18,19]$. In addition, when valuating for the outcome of death, the meta-analysis included a study published as an abstract, which had a large impact on the results $[17,20]$. The authors did not carry out sensitive analysis to determine the stability of the results [17]. In addition, Cochrane reviews also have compared inhaled and systemic corticosteroids [19, 21, 22]. Most of the main outcome were concluded from a small number of the studies with few infants providing data [21, 22].

Uncertainty prevails with regard to the use of inhalation or instillation steroids to prevent BPD in preterm infants [23]. Recently, 2 RCTS about airway administration of corticosteroids (AACs) for preventing BPD and neurodevelopmental outcomes have been published [24, 25]. Therefore, to better understand the potential efficacy and safety of AACs for reducing the risk of BPD or/and death in preterm infants, as compared with placebo or systemic corticosteroids, we performed an up-to-date systematic review of published RCTs. We verified the robustness and reliability of our findings by implementing trial sequential analysis and sensitivity analyses.

\section{Methods}

\section{Study identification}

This systematic review was conducted and is reported according to the recommendations of the Preferred Reporting Items for Systematic Reviews and Meta-Analyses (PRISMA) statement [26]. Electronic searches were carried out in multiple databases, including PubMed, Web of Science, Embase, Cochrane Library, Clinicaltrials.gov, Controlled-trials.com, Google scholar, VIP, WangFang and proceedings of the Pediatric Academic Society meetings (from 1980) on December 31, 2016 for relevant studies.
Search terms included: preterm infant, premature infant, infant-newborn, bronchopulmonary dysplasia, chronic lung diseases, anti-inflammatory agents, neurodevelopmental outcomes, steroids, glucocorticoids, corticosteroids; administration, inhalation; aerosols, budesonide, beclomethasone, dipropionate, flunisolide, and fluticasone propionate. No language restriction was applied. The protocol of this systematic review was registered before the literature search in PROSPERO (Prospero2016 CRD42016054098) [27].

\section{Eligibility criteria}

Studies had to meet the following criteria: 1) randomized controlled trials; 2) infants were randomized to receive treatment with an airway administration (inhalation or instillation) of corticosteroid vs placebo or systemic corticosteroids; 3) reported more than one of the following outcome parameters: primary outcome of BPD (defined by the need for supplemental oxygen or positive pressure support at 36 weeks PMA) or/and death; secondary outcomes of the use of systemic corticosteroids, effect on extubation and not extubate (including extubated within 14 days and duration of mechanical ventilation), adverse outcomes of sepsis, hyperglycemia requiring treatment, intraventricular hemorrhage (IVH), periventricular leukomalacia (PVL), necrotizing enterocolitis (NEC) Bell's stage $>$ or $=$ II, retinopathy of prematurity (ROP), PDA requiring drug treatment or surgery, and neurodevelopmental outcomes of neurodevelopmental impairment and cerebral palsy. Exclusion criteria were: a) non-clinical studies (experimental and basic studies); b) observational or retrospective studies; c) duplicate reports or secondary or post hoc analyses of the same study population; d) lack of sufficient information on baseline or primary or secondary outcome data; and e) therapeutic study.

\section{Assessment of the risk of bias}

Two reviewers (Zhang and Zhong) independently assessed the risk of bias of individual studies and the bias domains across studies using the Cochrane collaboration tool [28]. All discrepancies were resolved by discussion and consensus. The studies were rated to be at high risk of bias, low risk of bias, or unclear risk of bias based on sequence generation, concealment of allocation, blinding of participants/parents and personnel, blinding of outcome assessment, incomplete outcome data, and selective outcome reporting.

\section{Data collection}

For each study, data were extracted independently by two reviewers (Zhang and Zhong) using a predesigned form. Any differences and disagreements in the abstracted data were discussed and resolved by consensus. Details of methodological quality, study design, analysis, and results 
were abstracted. For each outcome, the numeric results, the statistic used, and the $P$ value were abstracted. We contacted authors of the original reports to obtain further details when information regarding any of the above information was unclear.

\section{Statistical analysis}

The statistical analyses were performed by the DerSimonian and Laird method (random-effect model) using the Review Manager meta-analysis software (version 5.3, 2012; The Cochrane Collaboration, Copenhagen, Denmark). TSA viewer version $0.9 \beta$ was used for trial sequential analysis. Treatment effect estimates for all trials were calculated, expressed as typical relative risk (RR) for dichotomous outcomes and weighted mean difference (WMD) for continuous outcomes, all with a 95\% confidence interval. If the continuous measures were reported in median and inter-quartile range, mean and standard SD values were estimated using the method described by Wan et al. [29]. $P$ values of $\leq 0.05$ and RR point estimate $95 \%$ CIs that excluded the null $(<1.00$ or $>1.00)$ were considered statistically significant. Where the pooled estimates of relative risk were statistically significant. We calculated the numbers needed to treat (NNT) for all outcomes. The heterogeneity between-trial regarding treatment effects was analyzed by the $X^{2}$ test for heterogeneity and $I^{2}$ statistics. Heterogeneity was deemed significant when the corresponding $p$-value was $<0.1$ or when the $I^{2}$ percentage was $>50$ [30]. Subgroup analyses were carried out to assess the source of heterogeneity. When more than 10 articles were included, the presence of publication bias was assessed and displayed through a funnel plot. Analysis by excluding studies at high risk of bias or abstract forms was part of a predefined subgroup analysis [28]. Sensitivity analysis with trial sequential analysis was performed to correct for random error and repetitive testing of accumulating and sparse data; meta-analysis monitoring boundaries and required information size (meta-analysis sample size) were quantified, along with D2 (diversity adjusted information size) and adjusted 95\% CIs [31-34]. Risk of type 1 error was considered $5 \%$ with a power of $80 \%$. A clinically meaningful anticipated relative risk reduction was used based on the low bias trial [16]. Trial sequential analysis 95\% CI boundaries that excluded the null $(<1.00$ or $>1.00)$ were considered statistically significant.

\section{Results}

\section{Study selection}

Our search identified 121 potentially relevant articles during the initial electronic database search, of which 35 RCTs were involving the use of AACs in premature infants. However, only 25 (27 articles) of 35 trials presented appropriate and sufficient data for further analysis [16, 20, $24,25,35-57]$, and the remaining eight were excluded because of therapeutic study of inhaled steroids for BPD $[18,19,58-61]$ and when the raw data in Ref. $[62,63]$ is lacking due to unable to reach the original investigators. Overall, the 20 trials of airway administration of corticosteroid (16 trials of inhaled corticosteroid and 4 trials of instillation of steroids) vs placebo included 2484 infants, and 5 trials of inhaled corticosteroid vs systemic corticosteroids included 765 infants. Two studies were published in abstract form [20, 47]. Two articles [50, 55] were follow-up studies of two included trials [49, 54]. Fig. 1 shows the details of the selection process.

\section{Characteristics of the included studies}

The 25 RCTs (27 articles) selected for analysis included a total of 3249 participants (Tables 1 and 2, Table S1-S2 in Additional file 1: Table S1 and Additional file 2: Table S2) $[16,20,24,25,35-57]$. The publication dates of the RCTs ranged from 1993 to 2016 . The AACs group vs the placebo group or systemic corticosteroids group were well matched; birth weight and gestational age did not differ significantly significance. Other aspects of respiratory treatment, including the resuscitation devices used and the criteria for using antenatal glucocorticoids as well as surfactant, were adequately described in the studies and conformed to current international guidelines [64-66]. The incidence of neonatal respiratory distress syndrome (was diagnosed based on respiratory symptoms and corresponding X-ray changes) was comparable among the airway administration of corticosteroid group and comparative groups.

Although all studies attempted to include infants at risk of developing $\mathrm{BPD}$, the inclusion criteria, the intervention (type of AACs), and duration of therapy varied between studies. The intervention regimens, including the prescribed dosages and administration schedules, varied considerably between the RCTs. Overall, the duration of AACs treatment ranged from 3 to 28 days, and the types of AACs included beclomethasone, budesonide, fluticasone, and dexamethasone. Delivery systems included metered dose inhaler (MDI) with a spacer device, nebulization, and airway instillation. Four studies used airway instillation of budesonide using surfactant as a vehicle [25, 36, 48, 49].

\section{Risk of bias within individual studies}

Three studies were deemed to have a low risk of bias [16, $24,25]$, seven studies were deemed to have a high risk of bias [36, 38, 53-57] due to selection bias, detection bias, attrition bias or performance bias, and 17 studies were classified as having an unclear risk of bias [20, 35, 37, 3952]. Although all studies were presented as randomized trials, the method of randomization was determined to be inadequate in 17 studies [20, 35, 37, 39-48, 51, 53, 56, 57]. Sixteen studies were found to have adequate concealment of allocation and clearly described blinding for the intervention method $[16,20,24,25,38,39,41,42,44,45,47$, 


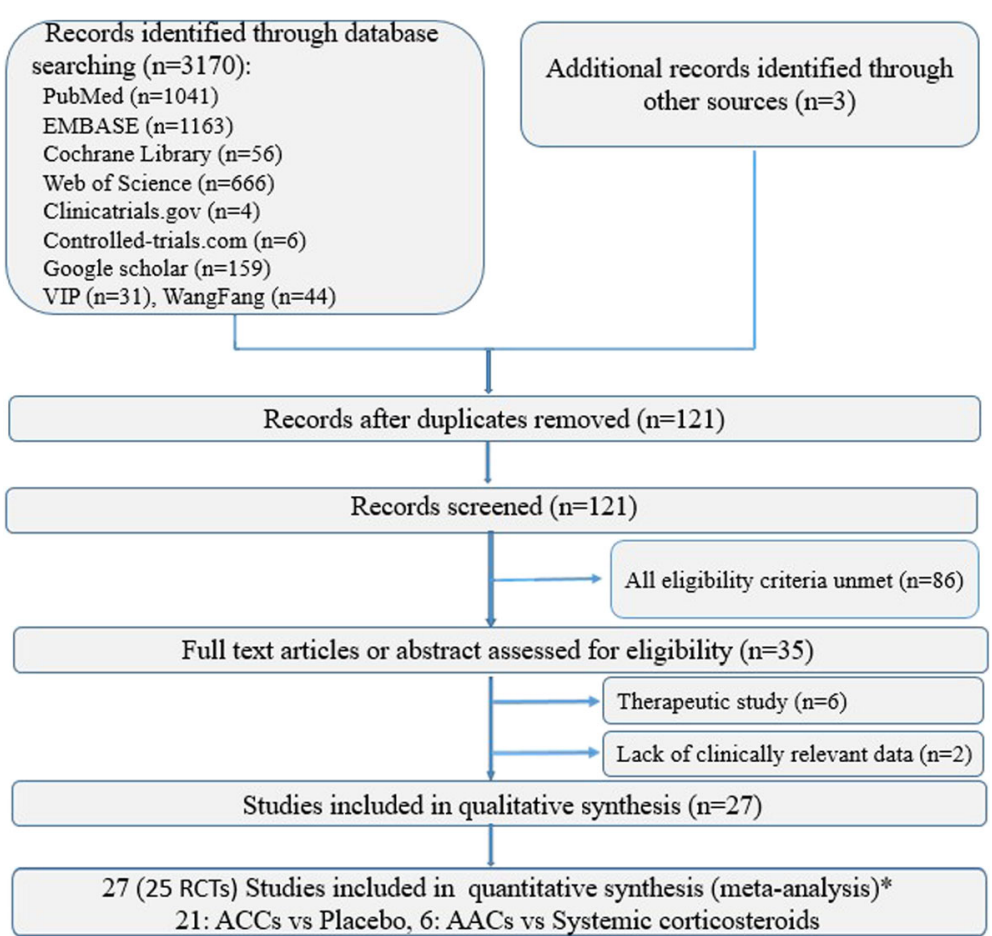

Fig. 1 Flowchart of the study selection process. * Two article [48,53] were follow-up study of two included trials [47, 52]

49-51, 56]. Thirteen studies were found to have adequate blinding of outcome assessment [16, 24, 25, 35, 37, 38, 40, $44,46,49,50,52,56]$. Follow-up data were reported in five studies $[24,25,41,50,56]$. Fig. 2 and 3 summarise the risks of bias.

\section{Systematic review of the findings from synthesis of the results}

\section{ACCs vs placebo \\ Primary clinical outcome \\ BPD}

Fourteen trials enrolling 2388 neonates reported on the incidence of BPD. Meta-analysis indicated that AACs was associated with a lower likelihood of BPD than was placebo $\left(\mathrm{RR}=0.71,95 \%\right.$ CI 0.58 to $0.86, \mathrm{NNT}=10, I^{2}=34 \%, P=$ 0.0005 ) (Fig. 4). Trial sequential analysis correction of the $95 \%$ confidence interval $\left(0.57\right.$ to $\left.0.87 ; \mathrm{D}^{2}=53 \%\right)$ did not alter the finding of BPD morbidity benefit with AACs (Fig.5). The finding was robust to sensitivity analysis (highest $P$ value 0.007 ) and the subgroup of instillation of steroids had the lowest NNT (Table 3), and clear evidence of publication bias was not present (Figure S1 in Additional file 3). Subgroup analysis based on type of corticosteroid showed that the incidence of BPD was significantly lower only in the group treated with budesonide compared to placebo $\left(\mathrm{RR}=0.59,95 \% \mathrm{CI} 0.44\right.$ to $0.79, \mathrm{NNT}=8, I^{2}=40 \%, P=$ 0.0004) (Fig. 4). In the trial sequential analysis, the cumulative $\mathrm{z}$ score crossed the sequential monitoring boundary of benefit and the required information size (Fig. 5), suggesting that further studies have little chance to perturbation the finding of benefit from AACs.

\section{Death}

Eleven trials enrolling 2041 neonates reported on the incidence of death. Meta-analysis indicated that the incidence of death was not significantly different between the AACs group and the placebo group ( $\mathrm{RR}=0.90,95 \% \mathrm{CI} 0.65$ to 1.25, $I^{2}=37 \%, P=0.55$ ) (Fig. 4). Trial sequential analysis correction of the $95 \%$ confidence interval (0.40 to 2.03; $\left.\mathrm{D}^{2}=55 \%\right)$ did demonstrate no increasing risk of death with airway administration of corticosteroid (Fig.5). The finding was robust to sensitivity analysis (Table 3), and clear evidence of publication bias was not present (Figure S1 in Additional file 3). Sensitivity analysis showed that instillation of steroids using surfactant as a vehicle had a nonsignificant reduction mortality $(12.1 \%$ vs. $17.8 \%$, $\mathrm{RR}=0.67 ; 95 \%$ CI 0.43 to $1.04, I^{2}=0 \%, P=0.07$ ) (Table 3 ).

\section{Death or BPD}

Eight trials enrolling 1761 neonates reported on the incidence of death or BPD. Meta-analysis indicated that airway administration of corticosteroid was associated with a lower likelihood of death or BPD than was placebo $\left(\mathrm{RR}=0.81,95 \% \mathrm{CI} 0.71\right.$ to 0.93 , NNT $=12, I^{2}=27 \%, P=$ 0.003) (Fig. 4). Trial sequential analysis correction of the 
Table 1 Characteristics of 25 RCTs reported in 27 articles and baseline characteristics of patients

\begin{tabular}{|c|c|c|c|c|c|c|c|c|c|}
\hline Study & $\begin{array}{l}\text { Study } \\
\text { characteristics }\end{array}$ & Group & $\mathrm{N}$ & $\begin{array}{l}\text { Male } \\
\text { (n) }\end{array}$ & GA (wk) & BW (g) & $\begin{array}{l}\text { Antenatal } \\
\text { glucocorticoid }\end{array}$ & $\operatorname{RDS}(n)$ & $\begin{array}{l}\text { Surfactant } \\
\text { (n) }\end{array}$ \\
\hline \multicolumn{10}{|l|}{ AACs vs placebo } \\
\hline Arnon [35] 1996 & $\begin{array}{l}1 \text { centre } \\
\text { UK }\end{array}$ & $\begin{array}{l}\mathrm{T} \\
\mathrm{C}\end{array}$ & $\begin{array}{l}15 \\
15\end{array}$ & $\begin{array}{l}\text { NA } \\
\text { NA }\end{array}$ & $\begin{array}{l}27.5(0.7)^{\mathrm{a}} \\
27.1(0.89)^{\mathrm{a}}\end{array}$ & $\begin{array}{l}1024(92.3)^{\mathrm{a}} \\
1041(113)^{\mathrm{a}}\end{array}$ & $\begin{array}{l}7(46.7 \%) \\
8(53.3 \%)\end{array}$ & $\begin{array}{l}\text { NA } \\
\text { NA }\end{array}$ & $\begin{array}{l}\text { NA } \\
\text { NA }\end{array}$ \\
\hline Bassler [16] 2015 & $\begin{array}{l}10 \text { centres } \\
\text { Switzerland }\end{array}$ & $\begin{array}{l}\mathrm{T} \\
\mathrm{C}\end{array}$ & $\begin{array}{l}437 \\
419\end{array}$ & $\begin{array}{l}\text { NA } \\
\text { NA }\end{array}$ & $\begin{array}{l}26.1(1.3)^{\mathrm{a}} \\
26.1(1.2)^{\mathrm{a}}\end{array}$ & $\begin{array}{l}798(193)^{\mathrm{a}} \\
803(189)^{\mathrm{a}}\end{array}$ & $\begin{array}{l}388(88.8 \%) \\
383(91.4 \%)\end{array}$ & $\begin{array}{l}\text { NA } \\
\text { NA }\end{array}$ & $\begin{array}{l}\text { NA } \\
\text { NA }\end{array}$ \\
\hline Cao [36] 2016 & $\begin{array}{l}1 \text { centre } \\
\text { China }\end{array}$ & $\begin{array}{l}\mathrm{T} \\
\mathrm{C}\end{array}$ & $\begin{array}{l}40 \\
40\end{array}$ & $\begin{array}{l}\text { NA } \\
\text { NA }\end{array}$ & $\begin{array}{l}30.1(2.2)^{\mathrm{a}} \\
30.7(1.8)^{\mathrm{a}}\end{array}$ & $\begin{array}{l}1333(110)^{\mathrm{a}} \\
1339(105)^{\mathrm{a}}\end{array}$ & $\begin{array}{l}\text { NA } \\
\text { NA }\end{array}$ & $\begin{array}{l}40 \\
40\end{array}$ & $\begin{array}{l}40 \\
40\end{array}$ \\
\hline Cole [37] 1999 & $\begin{array}{l}4 \text { centres } \\
\text { USA }\end{array}$ & $\begin{array}{l}\mathrm{T} \\
\mathrm{C}\end{array}$ & $\begin{array}{l}123 \\
130\end{array}$ & $\begin{array}{l}51 \\
53\end{array}$ & $\begin{array}{l}26(2)^{a} \\
26(2)^{a}\end{array}$ & $\begin{array}{l}800(193)^{\mathrm{a}} \\
802(189)^{\mathrm{a}}\end{array}$ & $\begin{array}{l}77(62.6 \%) \\
68(52.3 \%)\end{array}$ & $\begin{array}{l}123 \\
129\end{array}$ & $\begin{array}{l}95 \\
97\end{array}$ \\
\hline Denjean [38] 1998 & $\begin{array}{l}6 \text { centres } \\
\text { France }\end{array}$ & $\begin{array}{l}\mathrm{T} \\
\mathrm{C}\end{array}$ & $\begin{array}{l}43 \\
43\end{array}$ & $\begin{array}{l}26 \\
26\end{array}$ & $\begin{array}{l}27.6(1.5)^{\mathrm{a}} \\
27.8(1.6)^{\mathrm{a}}\end{array}$ & $\begin{array}{l}1060(218)^{a} \\
1082(260)^{a}\end{array}$ & $\begin{array}{l}11(25.6 \%) \\
11(25.6 \%)\end{array}$ & $\begin{array}{l}43 \\
43\end{array}$ & $\begin{array}{l}31 \\
34\end{array}$ \\
\hline Fok [39] 1999 & $\begin{array}{l}1 \text { centre } \\
\text { Hong Kong }\end{array}$ & $\begin{array}{l}\mathrm{T} \\
\mathrm{C}\end{array}$ & $\begin{array}{l}27 \\
26\end{array}$ & $\begin{array}{l}\text { NA } \\
\text { NA }\end{array}$ & $\begin{array}{l}27.9(0.5)^{\mathrm{a}} \\
27.1(0.5)^{\mathrm{a}}\end{array}$ & $\begin{array}{l}993(71)^{\mathrm{a}} \\
981(71)^{\mathrm{a}}\end{array}$ & $\begin{array}{l}15(55.6 \%) \\
13(50.0 \%)\end{array}$ & $\begin{array}{l}\text { NA } \\
\text { NA }\end{array}$ & $\begin{array}{l}\text { NA } \\
\text { NA }\end{array}$ \\
\hline Giep [40] 1996 & $\begin{array}{l}1 \text { centre } \\
\text { USA }\end{array}$ & $\begin{array}{l}\mathrm{T} \\
\mathrm{C}\end{array}$ & $\begin{array}{l}10 \\
9\end{array}$ & $\begin{array}{l}6 \\
3\end{array}$ & $\begin{array}{l}26(2.0)^{\mathrm{a}} \\
25(1.6)^{\mathrm{a}}\end{array}$ & $\begin{array}{l}752(110)^{\mathrm{a}} \\
784(141)^{\mathrm{a}}\end{array}$ & $\begin{array}{l}4(40.0 \%) \\
2(22.2 \%)\end{array}$ & $\begin{array}{l}8 \\
9\end{array}$ & $\begin{array}{l}8 \\
9\end{array}$ \\
\hline Jangaard [41] 2002 & $\begin{array}{l}1 \text { centre } \\
\text { Germany }\end{array}$ & $\begin{array}{l}\mathrm{T} \\
\mathrm{C}\end{array}$ & $\begin{array}{l}30 \\
30\end{array}$ & $\begin{array}{l}13 \\
13\end{array}$ & $\begin{array}{l}27.2(2)^{a} \\
27.9(2)^{a}\end{array}$ & $\begin{array}{l}882(204)^{a} \\
917(178)^{b}\end{array}$ & $\begin{array}{l}15(50.0 \%) \\
16(53.3 \%)\end{array}$ & $\begin{array}{l}19 \\
25\end{array}$ & $\begin{array}{l}19 \\
25\end{array}$ \\
\hline Jonsson [42] 2000 & $\begin{array}{l}1 \text { centre } \\
\text { Sweden }\end{array}$ & $\begin{array}{l}\mathrm{T} \\
\mathrm{C}\end{array}$ & $\begin{array}{l}15 \\
15\end{array}$ & $\begin{array}{l}8 \\
11\end{array}$ & $\begin{array}{l}25(23,27)^{b} \\
26(24,29)^{b}\end{array}$ & $\begin{array}{l}766(525,1122)^{b} \\
813(630,1227)^{b}\end{array}$ & $\begin{array}{l}12(80.0 \%) \\
10(66.7 \%)\end{array}$ & $\begin{array}{l}14 \\
15\end{array}$ & $\begin{array}{l}14 \\
15\end{array}$ \\
\hline $\begin{array}{l}\text { Ke [43] } \\
2016\end{array}$ & $\begin{array}{l}1 \text { centre } \\
\text { China }\end{array}$ & $\begin{array}{l}\mathrm{T} \\
\mathrm{C}\end{array}$ & $\begin{array}{l}46 \\
46\end{array}$ & $\begin{array}{l}20 \\
27\end{array}$ & $\begin{array}{l}<32 \\
<32\end{array}$ & $\begin{array}{l}<1500 \\
<1500\end{array}$ & $\begin{array}{l}\text { NA } \\
\text { NA }\end{array}$ & $\begin{array}{l}46 \\
46\end{array}$ & $\begin{array}{l}46 \\
46\end{array}$ \\
\hline LaForce [44] 1993 & $\begin{array}{l}1 \text { centre } \\
\text { USA }\end{array}$ & $\begin{array}{l}\mathrm{T} \\
\mathrm{C}\end{array}$ & $\begin{array}{l}10 \\
11\end{array}$ & $\begin{array}{l}\text { NA } \\
\text { NA }\end{array}$ & $\begin{array}{l}\text { NA } \\
\text { NA }\end{array}$ & $\begin{array}{l}<1500 \\
<1500\end{array}$ & $\begin{array}{l}\text { NA } \\
\text { NA }\end{array}$ & $\begin{array}{l}\text { NA } \\
\text { NA }\end{array}$ & $\begin{array}{l}\text { NA } \\
\text { NA }\end{array}$ \\
\hline Merz [45] 1999 & $\begin{array}{l}1 \text { centre } \\
\text { Germany }\end{array}$ & $\begin{array}{l}\mathrm{T} \\
\mathrm{C}\end{array}$ & $\begin{array}{l}12 \\
11\end{array}$ & $\begin{array}{l}5 \\
7\end{array}$ & $\begin{array}{l}28(27,32)^{\mathrm{b}} \\
29(27,31)^{\mathrm{b}}\end{array}$ & $\begin{array}{l}1108(820,1420)^{b} \\
1120(880,1480)^{b}\end{array}$ & $\begin{array}{l}7(58.3 \%) \\
7(63.6 \%)\end{array}$ & $\begin{array}{l}12 \\
11\end{array}$ & $\begin{array}{l}10 \\
9\end{array}$ \\
\hline Nakamura [24] 2016 & $\begin{array}{l}1 \text { centre } \\
\text { Japan }\end{array}$ & $\begin{array}{l}\mathrm{T} \\
\mathrm{C}\end{array}$ & $\begin{array}{l}107 \\
104\end{array}$ & $\begin{array}{l}63 \\
50\end{array}$ & $\begin{array}{l}26(25,27)^{\mathrm{b}} \\
26(25,27)^{\mathrm{b}}\end{array}$ & $\begin{array}{l}783.87(134.8)^{b} \\
784.06(127.2)^{b}\end{array}$ & $\begin{array}{l}45(42.1 \%) \\
42(40.4 \%)\end{array}$ & $\begin{array}{l}90 \\
88\end{array}$ & $\begin{array}{l}\text { NA } \\
\text { NA }\end{array}$ \\
\hline Pappagallo [46] 1998 & $\begin{array}{l}1 \text { centre } \\
\text { USA }\end{array}$ & $\begin{array}{l}\mathrm{T} \\
\mathrm{C}\end{array}$ & $\begin{array}{l}9 \\
9\end{array}$ & $\begin{array}{l}5 \\
5\end{array}$ & $\begin{array}{l}26.8(1.1)^{\mathrm{a}} \\
26.6(0.8)^{\mathrm{a}}\end{array}$ & $\begin{array}{l}828(64)^{a} \\
849(89)^{a}\end{array}$ & $\begin{array}{l}\text { NA } \\
\text { NA }\end{array}$ & $\begin{array}{l}\text { NA } \\
\text { NA }\end{array}$ & $\begin{array}{l}\text { NA } \\
\text { NA }\end{array}$ \\
\hline Townsend [47] 1998 & $\begin{array}{l}1 \text { centre } \\
\text { Denver }\end{array}$ & $\begin{array}{l}\mathrm{T} \\
\mathrm{C}\end{array}$ & $\begin{array}{l}15 \\
17\end{array}$ & $\begin{array}{l}8 \\
8\end{array}$ & $\begin{array}{l}25.8 \\
25.5\end{array}$ & $\begin{array}{l}728 \\
695\end{array}$ & $\begin{array}{l}\text { NA } \\
\text { NA }\end{array}$ & $\begin{array}{l}15 \\
17\end{array}$ & $\begin{array}{l}\text { NA } \\
\text { NA }\end{array}$ \\
\hline Wen [48] 2016 & $\begin{array}{l}1 \text { centre } \\
\text { China }\end{array}$ & $\begin{array}{l}\mathrm{T} \\
\mathrm{C}\end{array}$ & $\begin{array}{l}80 \\
80\end{array}$ & $\begin{array}{l}54 \\
51\end{array}$ & $\begin{array}{l}30.5(2.6)^{\mathrm{a}} \\
31.2(2.3)^{\mathrm{a}}\end{array}$ & $\begin{array}{l}1220(183)^{\mathrm{a}} \\
1250(167)^{\mathrm{a}}\end{array}$ & $\begin{array}{l}\text { NA } \\
\text { NA }\end{array}$ & $\begin{array}{l}\text { NA } \\
\text { NA }\end{array}$ & $\begin{array}{l}\text { NA } \\
\text { NA }\end{array}$ \\
\hline $\begin{array}{l}\text { Yeh [49] } 2008 \\
\text { Kuo [50] } 2010\end{array}$ & $\begin{array}{l}1 \text { centre } \\
\text { Taiwan }\end{array}$ & $\begin{array}{l}\mathrm{T} \\
\mathrm{C}\end{array}$ & $\begin{array}{l}60 \\
56\end{array}$ & $\begin{array}{l}31 \\
29\end{array}$ & $\begin{array}{l}26.4(2.2)^{\mathrm{a}} \\
26.7(2.3)^{\mathrm{a}}\end{array}$ & $\begin{array}{l}881(245)^{\mathrm{a}} \\
919(272)^{\mathrm{a}}\end{array}$ & $\begin{array}{l}46(76.7 \%) \\
42(75.0 \%)\end{array}$ & $\begin{array}{l}60 \\
56\end{array}$ & $\begin{array}{l}60 \\
56\end{array}$ \\
\hline Yeh [25] 2016 & $\begin{array}{l}1 \text { centre } \\
\text { Taiwan }\end{array}$ & $\begin{array}{l}\mathrm{T} \\
\mathrm{C}\end{array}$ & $\begin{array}{l}131 \\
134\end{array}$ & $\begin{array}{l}\text { NA } \\
\text { NA }\end{array}$ & $\begin{array}{l}26.5(2.2)^{a} \\
26.8(2.2)^{a}\end{array}$ & $\begin{array}{l}882(249)^{\mathrm{a}} \\
935(283)^{\mathrm{a}}\end{array}$ & $\begin{array}{l}\text { NA } \\
\text { NA }\end{array}$ & $\begin{array}{l}131 \\
134\end{array}$ & $\begin{array}{l}131 \\
134\end{array}$ \\
\hline Yong [20] 1999 & $\begin{array}{l}1 \text { centre } \\
\text { UK }\end{array}$ & $\begin{array}{l}\mathrm{T} \\
\mathrm{C}\end{array}$ & $\begin{array}{l}20 \\
20\end{array}$ & $\begin{array}{l}13 \\
12\end{array}$ & $\begin{array}{l}27.4(1.7)^{\mathrm{a}} \\
27.7(1.7)^{\mathrm{a}}\end{array}$ & $\begin{array}{l}1011(223)^{\mathrm{a}} \\
932(401)^{\mathrm{a}}\end{array}$ & $\begin{array}{l}\text { NA } \\
\text { NA }\end{array}$ & $\begin{array}{l}\text { NA } \\
\text { NA }\end{array}$ & $\begin{array}{l}\text { NA } \\
\text { NA }\end{array}$ \\
\hline Zimmerman [51] 2000 & $\begin{array}{l}1 \text { centre } \\
\text { USA }\end{array}$ & $\begin{array}{l}\mathrm{T} \\
\mathrm{C}\end{array}$ & $\begin{array}{l}20 \\
19\end{array}$ & $\begin{array}{l}12 \\
8\end{array}$ & $\begin{array}{l}26(2.0)^{\mathrm{a}} \\
26(2.0)^{\mathrm{a}}\end{array}$ & $\begin{array}{l}910(198)^{a} \\
802(225)^{a}\end{array}$ & $\begin{array}{l}14(70.0 \%) \\
15(78.9 \%)\end{array}$ & $\begin{array}{l}15 \\
18\end{array}$ & $\begin{array}{l}15 \\
18\end{array}$ \\
\hline \multicolumn{10}{|c|}{ Inhaled corticosteroids vs systemic corticosteroids } \\
\hline Dimitriou [52] 1997 & $\begin{array}{l}1 \text { centre } \\
\text { UK }\end{array}$ & $\begin{array}{l}\mathrm{T} \\
\mathrm{C}\end{array}$ & $\begin{array}{l}20 \\
20\end{array}$ & $\begin{array}{l}\text { NA } \\
\text { NA }\end{array}$ & $\begin{array}{l}27(24,30)^{b} \\
27(24,31)\end{array}$ & $\begin{array}{l}849(584,1270)^{b} \\
818(425,1460)^{b}\end{array}$ & $\begin{array}{l}12(60.0 \%) \\
11(55.0 \%)\end{array}$ & $\begin{array}{l}\text { NA } \\
\text { NA }\end{array}$ & $\begin{array}{l}14 \\
12\end{array}$ \\
\hline Groneck [53] 1999 & $\begin{array}{l}1 \text { centre } \\
\text { Germany }\end{array}$ & $\begin{array}{l}\mathrm{T} \\
\mathrm{C}\end{array}$ & $\begin{array}{l}7 \\
9\end{array}$ & $\begin{array}{l}3 \\
3\end{array}$ & $\begin{array}{l}26(25,28)^{b} \\
26(25,28)^{b}\end{array}$ & $\begin{array}{l}800(500,1020)^{b} \\
847(660,1030)^{b}\end{array}$ & $\begin{array}{l}\text { NA } \\
\text { NA }\end{array}$ & $\begin{array}{l}\text { NA } \\
\text { NA }\end{array}$ & $\begin{array}{l}\text { NA } \\
\text { NA }\end{array}$ \\
\hline $\begin{array}{l}\text { Halliday [54] } 2001 \\
\text { Wilson [55] } 2006\end{array}$ & $\begin{array}{l}47 \text { centre } \\
\text { Europe }\end{array}$ & $\begin{array}{l}\mathrm{T} \\
\mathrm{C}\end{array}$ & $\begin{array}{l}285 \\
285\end{array}$ & $\begin{array}{l}142 \\
164\end{array}$ & $\begin{array}{l}27.2(1.9)^{\mathrm{a}} \\
27.2(1.9)^{\mathrm{a}}\end{array}$ & $\begin{array}{l}1002(281)^{\mathrm{a}} \\
1011(285)^{\mathrm{a}}\end{array}$ & $\begin{array}{l}177(62.1 \%) \\
164(57.5 \%)\end{array}$ & $\begin{array}{l}\text { NA } \\
\text { NA }\end{array}$ & $\begin{array}{l}265 \\
260\end{array}$ \\
\hline Rozycki [56] 2003 & $\begin{array}{l}1 \text { centre } \\
\text { USA }\end{array}$ & $\begin{array}{l}\mathrm{T} \\
\mathrm{C}\end{array}$ & $\begin{array}{l}46 \\
15\end{array}$ & $\begin{array}{l}23 \\
7\end{array}$ & $\begin{array}{l}<31 \\
<31\end{array}$ & $\begin{array}{l}<1500 \\
<1500\end{array}$ & $\begin{array}{l}6(13.0 \%) \\
2(13.3 \%)\end{array}$ & $\begin{array}{l}46 \\
15\end{array}$ & $\begin{array}{l}46 \\
15\end{array}$ \\
\hline Suchomski [57] 2002 & $\begin{array}{l}1 \text { centre } \\
\text { USA }\end{array}$ & $\begin{array}{l}\mathrm{T} \\
\mathrm{C}\end{array}$ & $\begin{array}{l}51 \\
27\end{array}$ & $\begin{array}{l}25 \\
19\end{array}$ & $\begin{array}{l}26(1.6)^{a} \\
26(2.0)^{a}\end{array}$ & $\begin{array}{l}844(157)^{\mathrm{a}} \\
843(227)^{\mathrm{a}}\end{array}$ & $\begin{array}{l}34(66.7 \%) \\
16(59.3 \%)\end{array}$ & $\begin{array}{l}\text { NA } \\
\text { NA }\end{array}$ & $\begin{array}{l}\text { NA } \\
\text { NA }\end{array}$ \\
\hline
\end{tabular}

RCTs Randomized controlled trials, $A A C s$ Airway administration of corticosteroids, RDS Respiratory distress syndrome, PS Pulmonary surfactant, $A G$ Antenatal glucocorticoid, GA Gestational age, BW Brith weight, ${ }^{a}$ means \pm SD, ${ }^{b}$ median (25th, 75 th percentiles), NA: Not Applicated 
Table 2 Interventions used in the 25 RCTs

\begin{tabular}{|c|c|c|c|c|c|}
\hline Study & $\begin{array}{l}\text { Age at } \\
\text { enrollment }\end{array}$ & $\begin{array}{l}\text { Type of } \\
\text { AACS }\end{array}$ & Intervention dose & Mode of delivery & Intervention treatment \\
\hline \multicolumn{6}{|l|}{ AACs vs placebo } \\
\hline $\begin{array}{l}\text { Arnon [35] } \\
1996\end{array}$ & $\begin{array}{l}15(0.6)^{a} \\
14(0.5)^{a}\end{array}$ & BUD & $600 \mu \mathrm{g} \mathrm{BID}$ & MDI & $\begin{array}{l}7 \text { days or until the infant } \\
\text { had been extubated }\end{array}$ \\
\hline Bassler [16] 2015 & within $12 \mathrm{~h}$ & BUD & $\begin{array}{l}400 \mu \mathrm{g} Q 12 \mathrm{H} \text { for } \\
14 \text { days } \\
200 \mu \mathrm{g} \text { Q12H for } \\
15 \text { days }\end{array}$ & $\mathrm{MDI}$ & $\begin{array}{l}\text { Until extubation and no } \\
\text { oxygen dependent or } 32 \\
\text { weeks PMA }\end{array}$ \\
\hline Cao [36] 2016 & within $6 \mathrm{~h}$ & BUD & $250 \mu \mathrm{g}$ Q8H & Airway instillation & Until $\mathrm{FIO}_{2}<0.4$ or extubation \\
\hline Cole [37] 1999 & $\begin{array}{l}5.7(3.4)^{a} \\
5.4(2.9)^{a}\end{array}$ & BDP & $\begin{array}{l}40 \mu \mathrm{g} / \mathrm{kg} \text { for } 7 \text { days } \\
30 \mu \mathrm{g} / \mathrm{kg} \text { for } 7 \text { days } \\
15 \mu \mathrm{g} / \mathrm{kg} \text { for } 7 \text { days } \\
5-10 \mu \mathrm{g} / \mathrm{kg} \text { for } 7 \text { days }\end{array}$ & $\mathrm{MDI}$ & 28 days \\
\hline Denjean [38] 1998 & 10 days & $\mathrm{BDP}$ & $250 \mu \mathrm{g}$ Q6H & $\mathrm{MDI}$ & 28 days \\
\hline Fok [39] 1999 & $\begin{array}{l}\text { within } 24 \mathrm{~h} \text { of } \\
\text { birth }\end{array}$ & FPP & $\begin{array}{l}250 \mu \mathrm{g} \\
\mathrm{Q} 12 \mathrm{H}\end{array}$ & $\mathrm{MDI}$ & 14 days \\
\hline Giep [40] 1996 & 14 days & $\mathrm{BDP}$ & $1 \mathrm{mg} / \mathrm{kg} / \mathrm{d}$ & $\mathrm{MDI}$ & 7 days \\
\hline $\begin{array}{l}\text { Jangaard [41] } \\
2002\end{array}$ & 3 years & $\mathrm{BDP}$ & $0.2 \mathrm{mg} / \mathrm{kg} / \mathrm{d}$ & $\mathrm{MDI}$ & Until 28 days of age \\
\hline Jonsson [42] 2000 & 6 days & BUD & $\begin{array}{l}0.5 \mathrm{mg} / \mathrm{times} \\
\text { BID }\end{array}$ & $\begin{array}{l}\text { Electronic dosimetric } \\
\text { jet nebulizer }\end{array}$ & 14 days \\
\hline Ke [43] 2016 & within $6 \mathrm{~h}$ & BUD & $250 \mu \mathrm{g} \mathrm{Q12H}$ & Airway instillation & Until stop respiratory support \\
\hline LaForce [44] 1993 & 14 days & $\mathrm{BDP}$ & $50 \mu \mathrm{g} \mathrm{Q8H}$ & $\begin{array}{l}\text { A Whisper Jet nebulizer } \\
\text { system }\end{array}$ & 28 days \\
\hline Merz [45] 1999 & 3 days & BUD & $1.6 \mathrm{mg} / \mathrm{d}$ Q6H & $\mathrm{MDI}$ & 10 days or until extubation \\
\hline $\begin{array}{l}\text { Nakamura [24] } \\
2016\end{array}$ & $\begin{array}{l}\text { within } 24 \mathrm{~h} \text { of } \\
\text { birth }\end{array}$ & FPP & $50 \mu \mathrm{g} \mathrm{BID}$ & $\mathrm{MDI}$ & Until 6 weeks of age or extubation \\
\hline $\begin{array}{l}\text { Pappagallo [46] } \\
1998\end{array}$ & $\begin{array}{l}22.6(3.0)^{a} \\
19.1(1.6)^{a}\end{array}$ & DXM & $\begin{array}{l}1 \mathrm{mg} / \mathrm{kg} \mathrm{Q} 8 \mathrm{H} \text { for } \\
7 \text { days } \\
0.5 \mathrm{mg} / \mathrm{kg} \text { Q8H for } \\
7 \text { days }\end{array}$ & A jet nebulizer & 10 days \\
\hline $\begin{array}{l}\text { Townsend [47] } \\
1998\end{array}$ & at $48-96 \mathrm{~h}$ of age & FPP & $500 \mu \mathrm{g}$ TID & MDI & 28 days or until extubation \\
\hline Wen [48] 2016 & $\begin{array}{l}\text { within } 24 \mathrm{~h} \text { of } \\
\text { birth }\end{array}$ & BUD & $\begin{array}{l}0.4 \mathrm{mg} / \mathrm{kg} \\
\mathrm{Q} 12 \mathrm{H}\end{array}$ & $\mathrm{MDI}$ & 14 days \\
\hline $\begin{array}{l}\text { Yeh [49] } 2008 \\
\text { Kuo [50] } 2010\end{array}$ & $2.1(2.2)^{\mathrm{a}}$ & BUD & $\begin{array}{l}0.25 \mathrm{mg} / \mathrm{kg} \\
\text { Q8H }\end{array}$ & Airway instillation & $\begin{array}{l}\text { Until the infant required } \leq 0.4 \text { of } \mathrm{FlO} 2 \\
\text { or until the infant was extubated }\end{array}$ \\
\hline $\begin{array}{l}\text { Yeh [25] } \\
2016\end{array}$ & $\begin{array}{l}2.0(1.5)^{a} \\
1.8(1.6)^{a}\end{array}$ & BUD & $\begin{array}{l}0.25 \mathrm{mg} / \mathrm{kg} \\
\mathrm{Q} 8 \mathrm{H}\end{array}$ & Airway instillation & $\begin{array}{l}\text { Until the infant required } \leq 0.4 \text { of } \\
\mathrm{FIO}_{2} \text { or until extubation }\end{array}$ \\
\hline $\begin{array}{l}\text { Yong [20] } \\
1999\end{array}$ & $\begin{array}{l}\text { within } 18 \mathrm{~h} \text { of } \\
\text { birth }\end{array}$ & FPP & $\begin{array}{l}250 \mu \mathrm{g} \\
\mathrm{Q} 12 \mathrm{H}\end{array}$ & $\mathrm{MDI}$ & 14 days \\
\hline $\begin{array}{l}\text { Zimmerman [51] } \\
2000\end{array}$ & $\begin{array}{l}\text { within } 24 \mathrm{~h} \text { of } \\
\text { birth }\end{array}$ & BDP & $\begin{array}{l}\text { 2MDI, Q6H for } 3 \text { days; } \\
\text { 2MDI, Q8H for } 3 \text { days; } \\
\text { 2MDI Q12H for } 3 \text { days; } \\
\text { 2MDI QD for } 3 \text { days }\end{array}$ & $\mathrm{MDI}$ & 12 days \\
\hline \multicolumn{6}{|c|}{ Inhaled corticosteroids vs systemic corticosteroids } \\
\hline $\begin{array}{l}\text { Dimitriou [52] } \\
1997\end{array}$ & After 5 days & BUD & $100 \mu \mathrm{g}$ QD & A jet nebulizer & 10 days \\
\hline $\begin{array}{l}\text { Groneck [53] } \\
1999\end{array}$ & After $72 \mathrm{~h}$ & BDP & $250 \mu \mathrm{g}$ QD & $\mathrm{MDI}$ & 10 28 days \\
\hline $\begin{array}{l}\text { Halliday [54] } 2001 \\
\text { Wilson [55] } 2006\end{array}$ & $<72 \mathrm{~h}$ or $>15 \mathrm{~d}$ & BUD & $\begin{array}{l}<1000 \mathrm{~g} 0.4 \mathrm{mg} \mathrm{BID} \\
>1000 \mathrm{~g} 0.6 \mathrm{mg} \mathrm{BID}\end{array}$ & $\mathrm{MDI}$ & 12 days or until extubation \\
\hline $\begin{array}{l}\text { Rozycki [56] } \\
2003\end{array}$ & at 14 days of age & $\mathrm{BDP}$ & $\begin{array}{l}\text { High: } 2.4-3.7 \mathrm{mg} / \mathrm{kg} / \mathrm{d} \\
\text { Mid: } 1.0-1.8 \mathrm{mg} / \mathrm{kg} / \mathrm{d}\end{array}$ & $\mathrm{MDI}$ & 7 days or until extubation \\
\hline
\end{tabular}


Table 2 Interventions used in the 25 RCTs (Continued)

\begin{tabular}{|c|c|c|c|c|c|}
\hline Study & $\begin{array}{l}\text { Age at } \\
\text { enrollment }\end{array}$ & $\begin{array}{l}\text { Type of } \\
\text { AACS }\end{array}$ & Intervention dose & Mode of delivery & Intervention treatment \\
\hline & & & Low: $0.5-0.7 \mathrm{mg} / \mathrm{kg} / \mathrm{d}$ & & \\
\hline $\begin{array}{l}\text { Suchomski [57] } \\
2002\end{array}$ & $\begin{array}{l}12-21 \text { days of } \\
\text { age }\end{array}$ & $\mathrm{BDP}$ & $\begin{array}{l}\text { High: } 800 \mu \mathrm{g} / \mathrm{d} \\
\text { Low: } 400 \mu \mathrm{g} / \mathrm{d}\end{array}$ & MDI & Until extubation \\
\hline
\end{tabular}

RCTs Randomized controlled trials, BUD Budesonide, BDP Beclomethasone dipropionate, FPP Fluticasone propionate, $M D I$ metered-dose inhaler, $A A C s$ Airway administration of corticosteroids, DXM Dexamethasone

None of the trials were sponsored by the manufacturer

${ }^{\mathrm{a}}$ means $\pm \mathrm{SD}$

$95 \%$ confidence interval $\left(0.71\right.$ to $\left.0.97 ; \mathrm{D}^{2}=55 \%\right)$ did support the finding of death or BPD morbidity benefit with airway administration of corticosteroid (Fig.5). The finding was robust to sensitivity analysis (highest $P$ value 0.03) (Table 3). Subgroup analysis based on type of corticosteroid showed that the incidence of death or BPD was significantly lower only in the group treated with budesonide versus placebo ( $\mathrm{RR}=0.77,95 \% \mathrm{CI} 0.61$ to 0.97, $\mathrm{NNT}=9, I^{2}=75 \%, P=0.03$ ) (Fig. 4).

\section{Secondary outcomes}

\section{Requirement for systemic steroids}

Fourteen trials enrolling 1886 neonates reported on the requirement for systemic steroids. A significant reduction in the administration of systemic corticosteroids was found in the group with airway administration of corticosteroids (RR $=0.86,95 \%$ CI 0.76 to 0.97, NNT $=20 I^{2}=1 \%, P=$ 0.02 ). However, publication bias was present (Figure S1 in Additional file 3). Subgroup analysis based on type of corticosteroid showed that the requirement for systemic steroids was significantly different only with beclomethasone versus placebo (Figure S2 in Additional file 4).

\section{Benefit to extubation}

Seven trials enrolling 552 neonates reported on rates of successful extubation within 14 days. AACs have a nonsignificant increasing rates of successful extubation within 14 days ( $55.1 \%$ vs. $44.6 \%, \mathrm{RR}=1.53,95 \%$ CI 1.0 to 2.33, $I^{2}=66 \%, P=0.05$ ) (Figure S3 in Additional file 5). Seven trials enrolling 623 neonates reported on the the duration of mechanical ventilation. AACs significantly reduce the duration of mechanical ventilation compared with placebo (WMD $=-2.99,95 \%$ CI -5.10 to $-0.87, I^{2}=$ $38 \%, P=0.006$ ) (Figure S4 in Additional file 6).

\section{Adverse outcomes}

AACs administration had a nonsignificant reduction in incidence of PDA $(40.2 \%$ vs. $45.2 \%, \mathrm{RR}=0.89,95 \% \mathrm{CI}$ 0.79 to $\left.1.0, I^{2}=0 \%, P=0.05\right)$ and NEC (7.3\% vs. $9.9 \%$, $\mathrm{RR}=0.75,95 \%$ CI 0.54 to $\left.1.04, I^{2}=0 \%, P=0.09\right)$ compared with placebo (Table S3 in Additional file 7). There were no significant differences between interventions in the likelihood of other adverse outcomes (including infection, hyperglycaemia, IVH, PVL, and ROP) (Table S3 in Additional file 7).

\section{Neurodevelopmental outcomes}

Four trials enrolling 474 neonates reported on the neurodevelopmental outcomes (neurodevelopmental impairment and cerebral palsy). Meta-analysis indicated that the incidence of neurodevelopmental impairment and cerebral palsy ware not significantly different between the airway administration of corticosteroid group and the placebo group (Table S3 in Additional file 7).

\section{Inhaled corticosteroids vs systemic corticosteroid Primary clinical outcome \\ Bpd}

Four trials enrolling 747 neonates reported on the incidence of BPD. Meta-analysis indicated that the morbidity

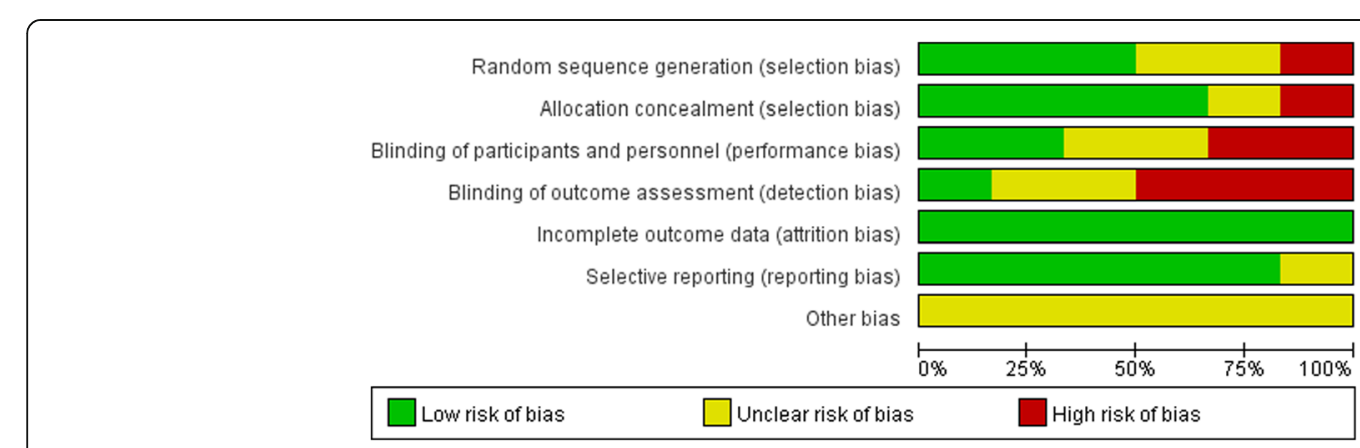

Fig. 2 Risk of bias graph 


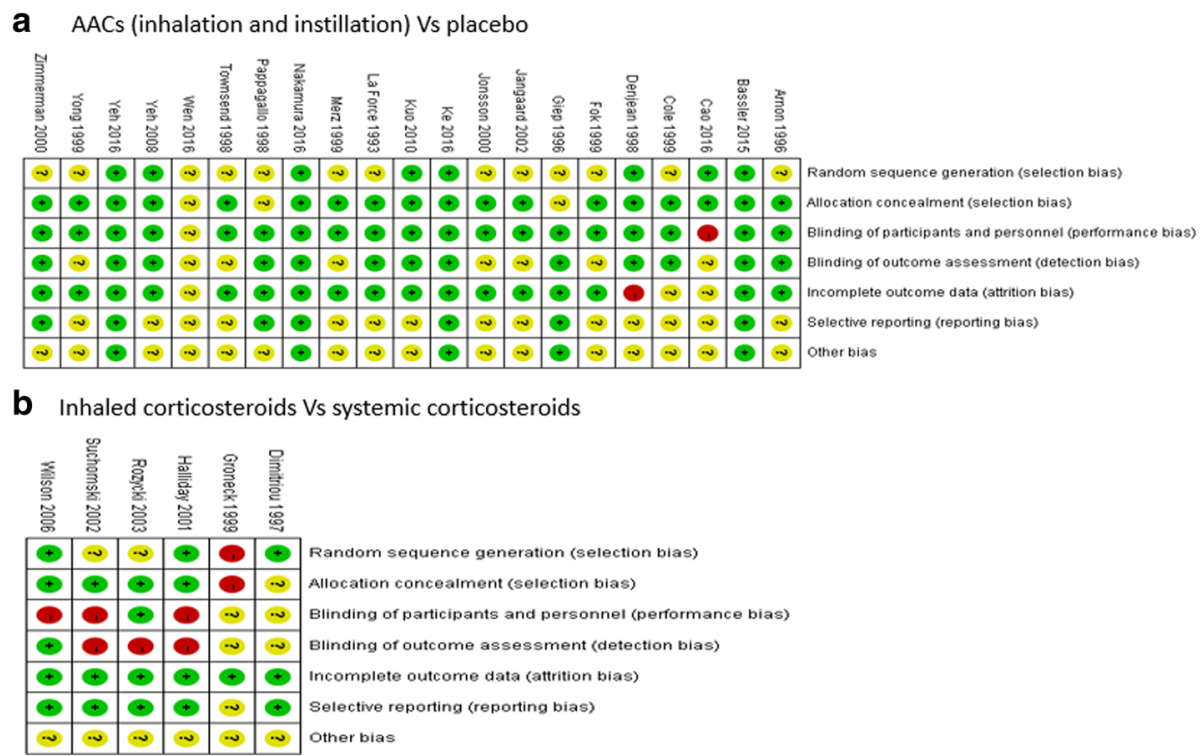

Fig. 3 Risk of bias summary for all included records

of BPD has no decisive difference between inhaled corticosteroid group and systemic corticosteroid group (RR = 1.02 , $95 \%$ CI 0.85 to $1.22, I^{2}=15 \%, P=0.81$ ) (Fig. 6). The finding was robust to trial sequential analysis $(R R=1.02$, adjusted $95 \%$ CI 0.55 to $1.88, D^{2}=0 \%, P=0.83$ ) (Fig. 7).

\section{Death}

Five trials enrolling 762 neonates reported on the incidence of death. Meta-analysis indicated that the mortality was not significantly different between the inhaled corticosteroid group and the systemic corticosteroid group (RR $=0.81,95 \%$ CI 0.62 to $1.06, I^{2}=0 \%, P=0.12$ ) (Fig. 6), and this finding was confirmed in trial sequential analysis (adjusted 95\% confidence interval 0.43 to 1.53). The cumulative $\mathrm{Z}$ curve did not cross any boundaries for benefit or harm (Fig. 7).

\section{Secondary outcomes \\ Benefit to extubation}

Four trials enrolling 693 neonates reported on the the duration of mechanical ventilation. Systemic corticosteroids were associated with shorter duration of mechanical ventilation $\left(\mathrm{WMD}=3.21,95 \% \mathrm{CI} 0.36\right.$ to $6.06, I^{2}=$ $10 \%, P=0.03$ ) (Figure S5 in Additional file 8).

\section{Adverse outcomes}

Inhaled corticosteroids were associated with less hyperglycemia ( $\mathrm{RR}=0.44,95 \% \mathrm{CI} 0.29$ to 0.69 , NNT $=9, I^{2}=0 \%, P=0.0003$ ) (Table S4 in Additional file 1). There were no significant differences between interventions in the likelihood of other adverse outcomes (including infection, NEC, PDA, PVL, and ROP) (Table S4 in Additional file 9).

\section{Neurodevelopmental outcomes}

One trial enrolling 126 neonates reported on the neurodevelopmental outcomes (impairment, disability, and cerebral palsy). There were no significant differences between interventions (Table S4 in Additional file 9).

\section{Discussion}

This meta-analysis including 25 trials with 3249 preterm infants at high risk of BPD estimated relative effects of AACs. The use of AACs was associated with a lower likelihood of the primary outcomes of BPD and death or BPD, and secondary outcomes of requirement for systemic steroids and the duration of mechanical ventilation than placebo, and was equivalent for preventing BPD than systemic corticosteroids. Moreover, AACs were not associated with an increased risk of death compared with placebo or systemic corticosteroids. AACs had less occurrence of hyperglycemia compared to systemic steroids.

\section{Interpretation of the findings}

Our primary outcomes, were robust to sensitivity and trial sequential analyses. When evaluating the incidence of BPD, death, and death or BPD between AACs and placebo, consistent results was observed in trial sequential analyses and after exclusion of studies at high risk of bias, small sample, non-English literature, abstract, or studies of instillation of steroids using surfactants as carriers. In addition, sensitivity analysis showed that NNT of 5 and 4 in subgroup of instillation of steroids using surfactant as a vehicle was lower than the NNT in the inhaled corticosteroids group $(\mathrm{NNT}=14$ and 20) for preventing one case of BPD and BPD or death (Table 3). Using as a vehicle surfactant may also enhance the 
a Bronchopulmonary dysplasia

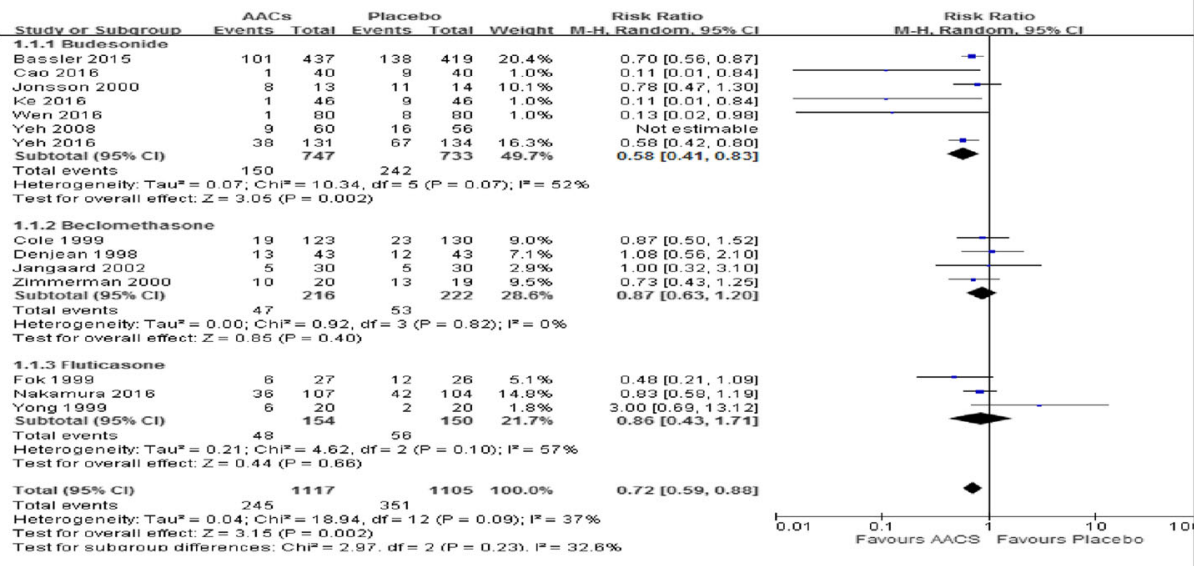

b Death

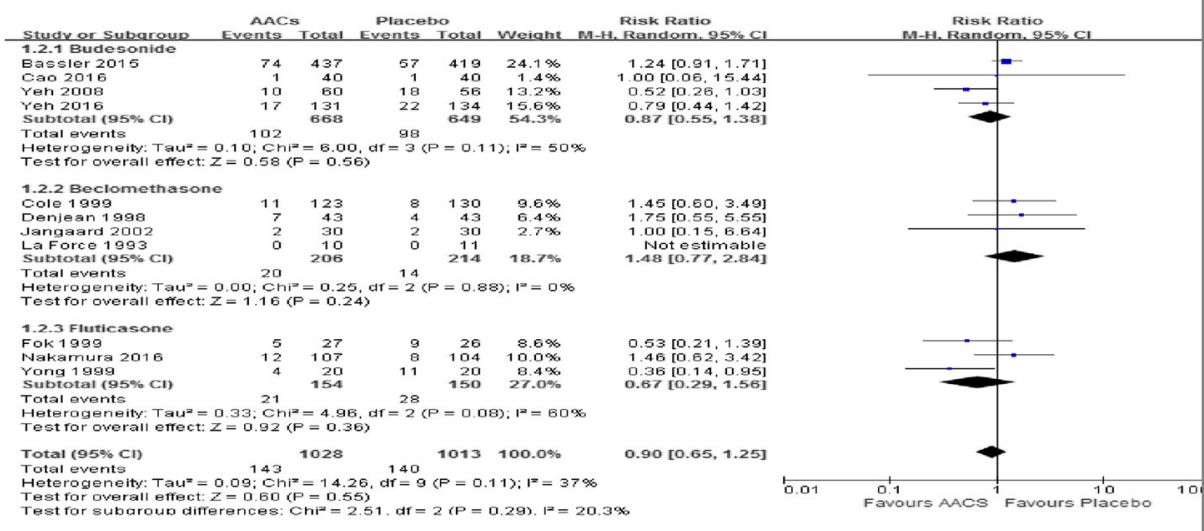

C Death or bronchopulmonary dysplasia

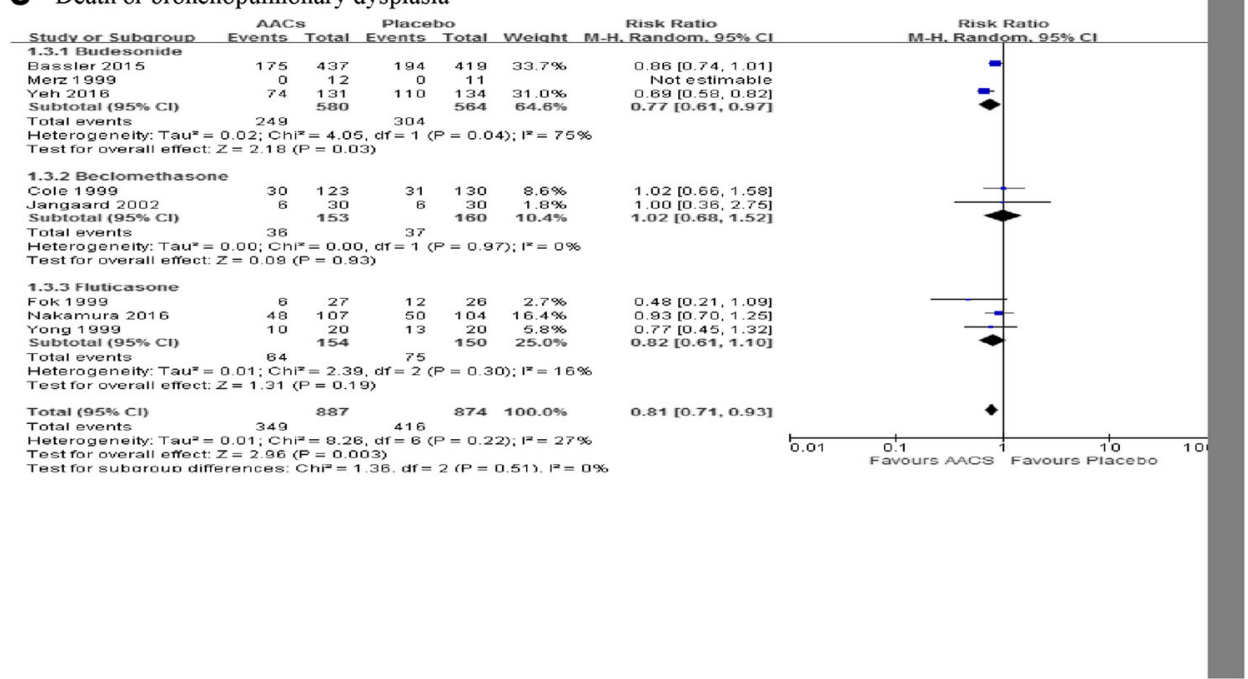

Fig. 4 Meta-analysis of primary outcome with the use of AACs or placebo, AACs: Airway administration of corticosteroids 
a Bronchopulmonary dysplasia

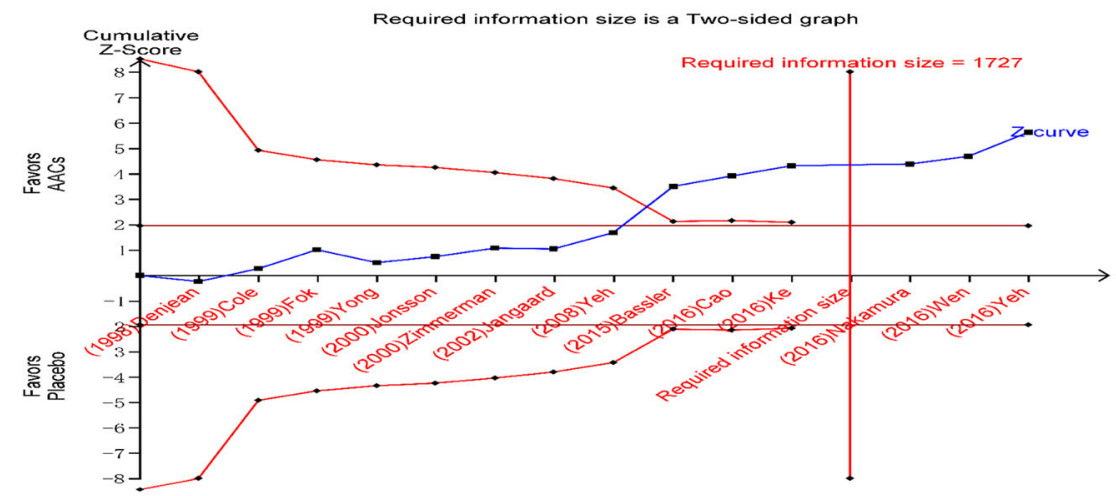

b Death

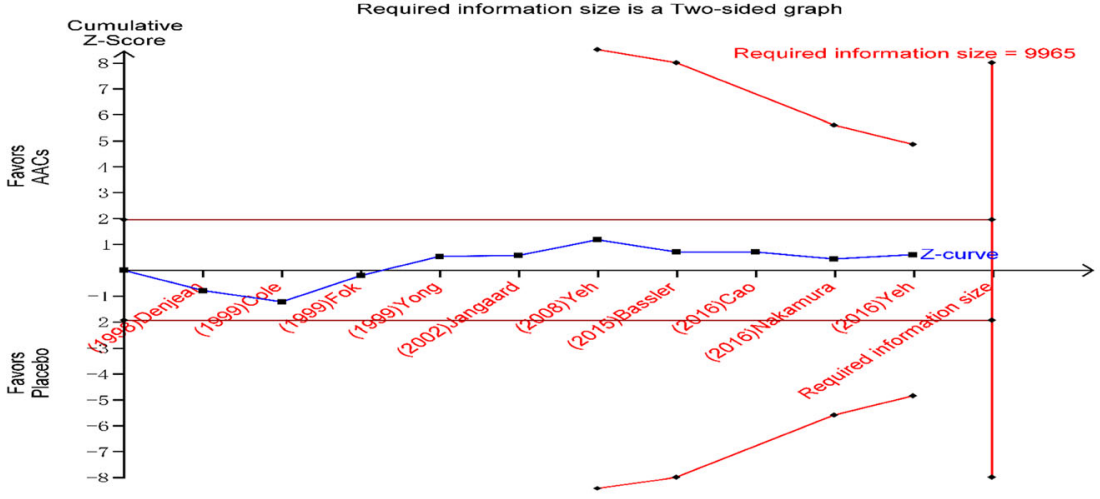

C Death or bronchopulmonary dysplasia

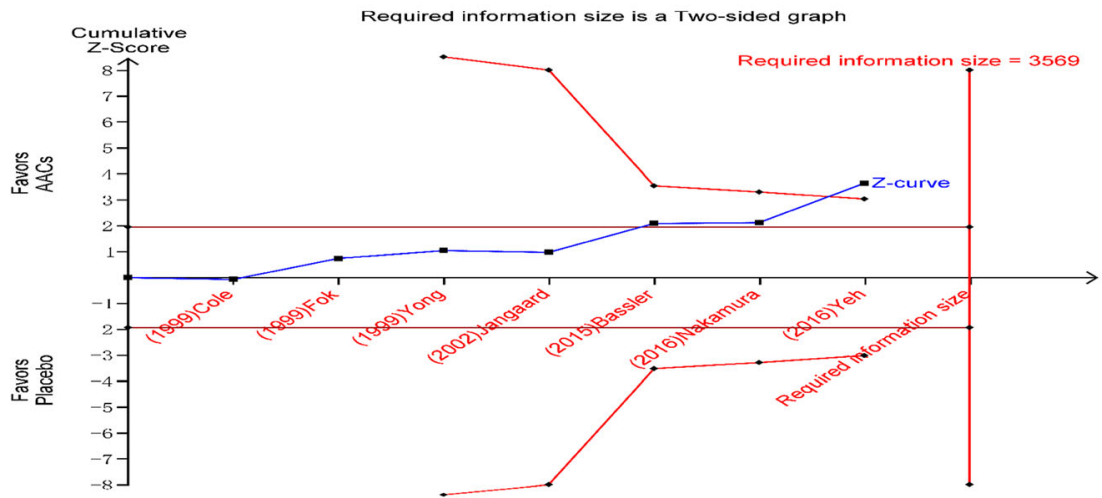

Fig. 5 (See legend on next page.) 
(See figure on previous page.)

Fig. 5 Trial sequential analysis of trials reporting primary outcome comparing pooled AACs and placebo. A diversity adjusted information size was calculated using $a=0.05$ (two sided), $\beta=0.20$ (power $80 \%$ ), D2 $=0 \%$, an anticipated relative risk of $29.8 \%,-24.5 \%$, $13.5 \%$, respectively, and an event proportion of $28.2 \%, 11.4 \%, 46.3 \%$, respectively in the control arm. The cumulative $z$ curve was constructed using a random-effect model, and a penalized $z$ curve was also constructed. The adjusted relative risk was $0.71,0.90,0.81$, respectively, and the $95 \%$ confidence interval was corrected to $0.57-0.87,0.40-2.03$, and 0.71-0.97, respectively, AACs: Airway administration of corticosteroids

solubility of budesonide, increase budesonide absorption, and strengthen the function of anti-inflammatory effects $[25,67]$. Trial sequential analysis corrected the $95 \%$ CIs of the already statistically significant point estimates for main comparison of an airway administration of corticosteroid with the placebo through accounting for random error and repetitive testing of accumulating sparse data. In our analysis of incidence of BPD and death or BPD between AACs and placebo, the cumulative $\mathrm{z}$ score entered the futility area, indicating further trials are not required. Considering the overall morbidity, no association with benefit or harm can be found between groups, but the trial sequential analysis suggested it would be unnecessary to carry out more trials on this outcome. In our analysis of neurodevelopmental outcome between AACs and placebo, AACs did not increase neurodevelopmental impairment. Futhermore, inhaled corticosteroid also did not increase mortality and is similar in preventing BPD compared with systemic corticosteroids. The information size for the comparison of inhaled corticosteroids with systemic corticosteroids was too low to require futility boundaries in trial sequential analysis. European Consensus Guidelines on the Management of Respiratory Distress Syndrome do not recommend the use of inhaled corticosteroid for preventing BPD until further safety data become available [68]. The benefit of preventing BPD and death or BPD associated with AACs found in this systematic review could inform future updates of these and other clinical guidelines.

\section{Comparison with other studies}

Several systematic reviews have evaluated inhaled corticosteroids in the prevention of BPD $[14,15,17,21$, 22]. A recent meta-analysis aimed to assess inhaled corticosteroids for BPD in preterm infants [17]. The evidence from this meta-analysis supported inhaled

Table 3 Sensitivity analysis of primary outcomes with the use of AACs or placebo

\begin{tabular}{|c|c|c|c|c|c|}
\hline Outcome or Subgroup & Studies & Participants & $P$ value & Effect Estimate & NNT \\
\hline$\overline{B P D}$ & 14 & 2338 & 0.0005 & $0.71[0.58,0.86]$ & 10 \\
\hline Exclusion of high risk trials & 12 & 2172 & 0.0001 & $0.70[0.58,0.84]$ & 10 \\
\hline Exclusion of conference abstracts & 13 & 2298 & $<0.0001$ & $0.69[0.58,0.83]$ & 9 \\
\hline Exclusion of non English studies & 11 & 2006 & $<0.00001$ & $0.72[0.63,0.83]$ & 10 \\
\hline Exclusion of small sample trials & 6 & 1861 & $<0.0001$ & $0.69[0.57,0.83]$ & 10 \\
\hline Inhaled corticosteroids & 10 & 1785 & 0.003 & $0.77[0.65,0.91]$ & 14 \\
\hline $\begin{array}{l}\text { Instillation of steroids using } \\
\text { surfactants as carriers }\end{array}$ & 4 & 553 & 0.007 & $0.44[0.24,0.79]$ & 5 \\
\hline Death & 11 & 2041 & 0.55 & $0.75[0.54,1.04]$ & \\
\hline Exclusion of high risk trials & 9 & 1875 & 0.40 & $0.88[0.56,1.39]$ & \\
\hline Exclusion of conference abstracts & 10 & 2003 & 0.99 & $1.00[0.75,1.33]$ & \\
\hline Exclusion of non English studies & 10 & 1961 & 0.55 & $0.90[0.64,1.27]$ & \\
\hline Exclusion of small sample trials & 5 & 1701 & 0.98 & $1.01[0.70,1.45]$ & \\
\hline Inhaled corticosteroids & 8 & 1580 & 0.88 & $1.03[0.69,1.53]$ & \\
\hline $\begin{array}{l}\text { Instillation of steroids using } \\
\text { surfactants as carriers }\end{array}$ & 3 & 461 & 0.07 & $0.67[0.43,1.04]$ & \\
\hline Death or BPD & 8 & 1761 & 0.003 & $0.81[0.71,0.93]$ & 12 \\
\hline Exclusion of conference abstracts & 7 & 1721 & 0.01 & $0.82[0.70,0.96]$ & 12 \\
\hline Exclusion of small sample trials & 4 & 1585 & 0.03 & $0.83[0.70,0.99]$ & 13 \\
\hline Inhaled corticosteroids & 7 & 1496 & 0.03 & $0.87[0.77,0.99]$ & 20 \\
\hline $\begin{array}{l}\text { Instillation of steroids using } \\
\text { surfactants as carriers }\end{array}$ & 1 & 265 & $<0.0001$ & $0.69[0.58,0.82]$ & 4 \\
\hline
\end{tabular}




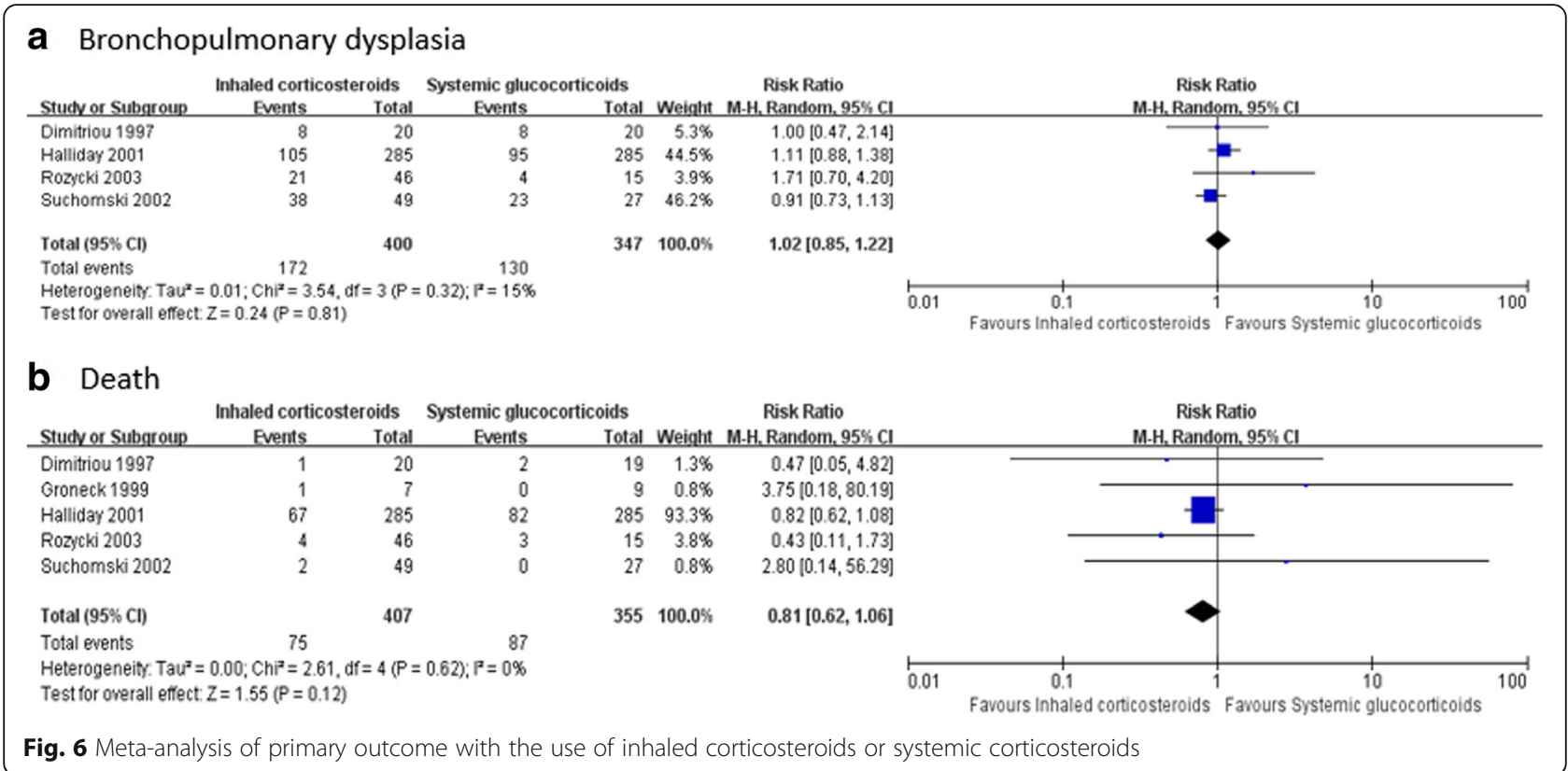

corticosteroids, especially budesonide, as a potentially efficacious and safe therapy for the prevention or treatment of BPD in preterm infants [17]. However they fail to implement sensitivity and subgroup analysis for the result of heterogeneity. In addition, they did not evaluate the advantage on inhaled corticosteroids compared with sysetmic corticosteroids. The previous Cochrane reviews examined either inhalation or systemic corticosteroids, further divided into early and late phases according to the time of administration. Hence, the analysis used 1 week after birth as a boundary [21, 22] However, in our meta-analysis, the duration of inhalation of corticosteroids ranged from 3 to 29 days, which was different from studies on intravenous administration, for which the course was about 1 week. For a long course of AACs, the boundary of 1 week after birth is sufficient to reflect the differences of AACs. Studies set the first delivery time from $12 \mathrm{~h}$ to 14 days after birth and the time span was long (Table 1). Therefore, we hypothesize that the subgroup analysis with 1 week after birth as the boundary is not reliable, and it is difficult to follow. The dose and duration of the AACs in each study were not the same, making it difficult to determine the exact dose and the course of corticosteroids that is supposed to be beneficial even via subgroup analysis. We believe that a unified drug delivery time and course of treatment are very important for developing precise treatment and firm recommendations.

\section{Strengths and limitations of this review}

The results of our analysis confirm those of the previous systematic reviews while improving their precision and further reducing the role of contingency. A major strength of present systematic review is the use of the robust Cochrane methodology [26, 28, 30], and the meta-analysis is further challenged with trial sequential analysis to correct for random error and repetitive testing, which often is biased towards an intervention [3133]. Our meta-analysis did focuse on bias and quality of evidence of included studies in accordance with GRADE [69]. Predefined sensitivity, subgroup analysis, and trial sequential analyses that included assessment of bias and clinically heterogenous groups were presented to aid healthcare professionals for clinical decisions. However, subtle underlying bias of the trials included in the review remains a possible limitation, as in any other systematic review although we excluded the studies that are at high risk of bias.

Admittedly, several limitations in our meta-analysis might have affected the interpretation of findings. First, the trials analyzed differed in their study design and clinical characteristics of the study participants. The effect on mortality and BPD might be quite different between very preterm infants (GA: 28 32 weeks) and extremely preterm infants $(\mathrm{GA}<28$ weeks), and subgroup analysis based on GA or BW could not be done due to the lack of individual patient data. For extremely preterm infants, the BPD definition is based on respiratory support and oxygen requirements at 36 weeks, which may be dependent on local practices and saturation targets. Despite attempts to standardize the definition of BPD, wide variation among centers has been reported with the diagnosis of BPD ranging from 6 to $57 \%$ depending on the definition chosen [70]. Hence the target population in each studies may be very 

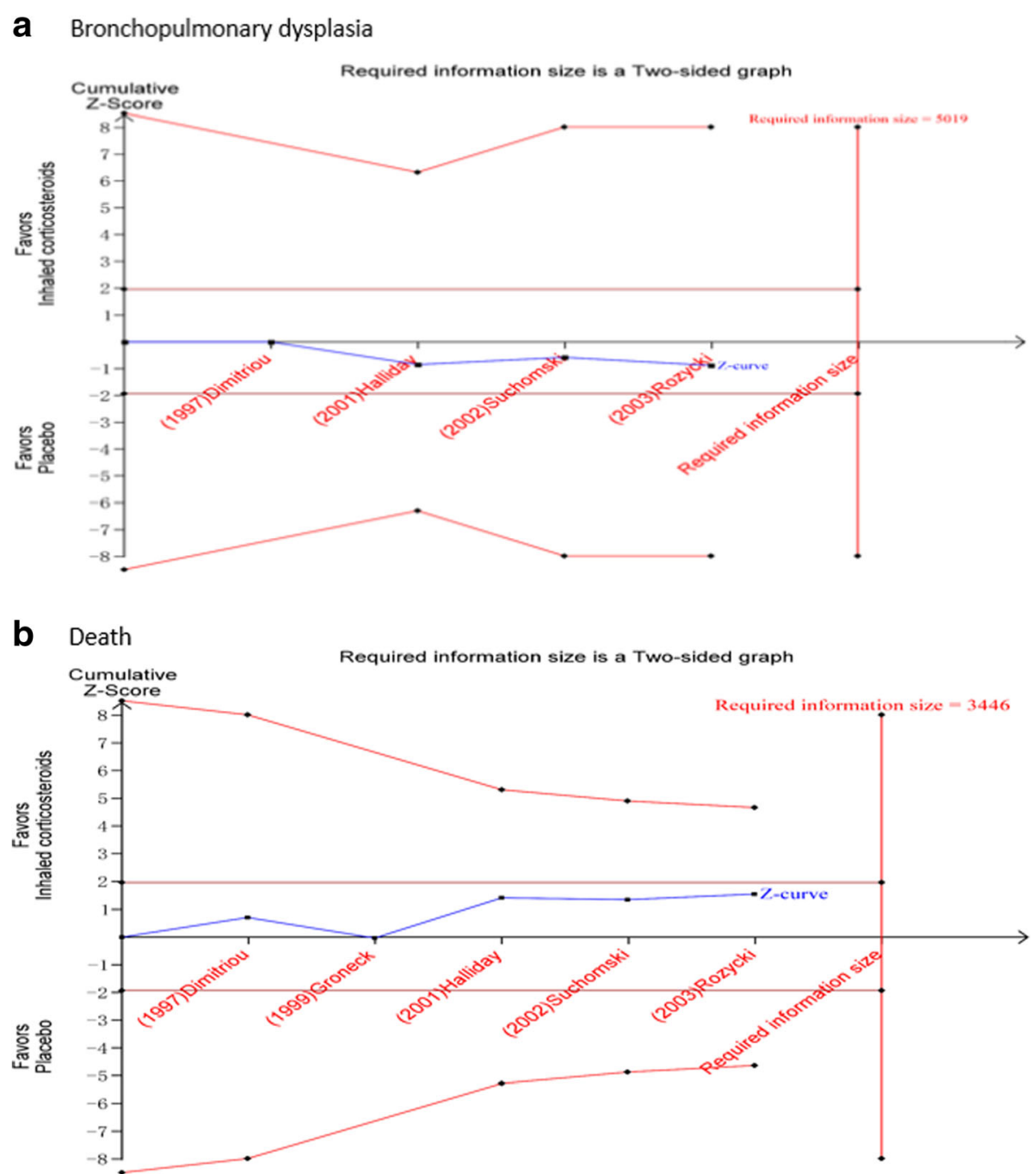

Fig. 7 Trial sequential analysis of trials reporting primary outcome comparing pooled inhaled corticosteroids and systemic corticosteroids. A diversity adjusted information size was calculated using $a=0.05$ (two sided), $\beta=0.20$ (power $80 \%$ ), D2 $=0 \%$, an anticipated relative risk of $-10.5 \%$, $18.4 \%$, respectively, and an event proportion of $36.6 \%, 20.0 \%$, respectively in the control arm. The cumulative $z$ curve was constructed using a random-effect model, and a penalized z curve was also constructed. The adjusted relative risk was $1.08,0.81$, respectively, and the $95 \%$ confidence interval was corrected to $0.53-2.20,0.43-1.53$, respectively

different. The duration, dose, type, and inhalation or instillation of steroids using surfactants as carriers also were inconsistent across studies. Although we validated the stability of the result of our meta-analysis by subgroup analysis and sensitivity, the direct comparison between inhalation and instillation steroids using surfactant as a vehicle was lacking. Furthermore, there were few studies on AACs compared with systemic corticosteroids, and not all trials presented the primary and secondary outcomes, be especially neurodevelopmental outcomes. To some extent, the clinical diversity still potentially compromises the validity of the current results, even though we have pooled the data as if they were from clinically homogeneous studies. Third, formal tests for publication bias are still lacking. In principle, this should not interfere with the metaanalysis results, because publication bias generally results in an overestimation of the effect estimates.

\section{Conclusions}

In this meta-analysis with sequential analysis, compared with placebo, AACs (especially instillation of budesonide using surfactant as a vehicle) was found to reduce the risk of BPD and death or BPD, with no assocaited increase in mortality and neurodevelopmental impairment. Compared with systemic corticosteroids, inhaled corticosteroids were similar for preventing BPD. The appropriate dose and duration, inhalation or instillation with surfactant as a vehicle and the long-term safety of AACs needs to be assessed in large trials. 


\section{Additional files}

Additional file 1: Table S1. Outcomes measured in the $25 \mathrm{RCTS}$ (DOCX $27 \mathrm{~kb}$ )

Additional file 2: Table S2. Outcomes measured in the $25 \mathrm{RCTS}$ (DOCX 29 kb)

Additional file 3: Figure S1. Funnel plot of bronchopulmonary dysplasia, death, and requirement for systemic steroids with the use of AACs or placebo, AACs: Airway administration of corticosteroids. (JPEG $50 \mathrm{~kb}$ )

Additional file 4: Figure S2. Meta-analysis of requirement for systemic steroids with the use of AACs or placebo, AACs: Airway administration of corticosteroids. (JPEG $26 \mathrm{~kb}$ )

Additional file 5: Figure S3. Meta-analysis of success to extubate within 14 days with the use of AACs or placebo, AACs: Airway administration of corticosteroids. (JPEG $20 \mathrm{~kb}$ )

Additional file 6: Figure S4. Meta-analysis of duration of mechanical ventilation with the use of AACs or placebo, AACs: Airway administration of corticosteroids. (JPEG $17 \mathrm{~kb}$ )

Additional file 7: Table S3. Subgroup analysis of adverse and neurodevelopmental outcomes with the use of AACs or placebo (DOCX $17 \mathrm{~kb}$ )

Additional file 8: Figure S5. Meta-analysis of duration of mechanical ventilation with the use of inhaled corticosteroids or systemic corticosteroids. (JPEG $11 \mathrm{~kb}$ )

Additional file 9: Table S4. Subgroup analysis of adverse and neurodevelopmental outcomes with the use of inhaled corticosteroids or systemic corticosteroids. (DOCX $15 \mathrm{~kb}$ )

\section{Abbreviations}

AACs: Airway administration of corticosteroids; BPD: Bronchopulmonary dysplasia; IVH: Intraventricular hemorrhage; MDI: Metered dose inhaler; NEC: Necrotizing enterocolitis; NNT: Number needed to treat; PDA: Patent ductus arteriosus; PMA: Postmenstrual age; PVL: Periventricular leukomalacia; RCTs: Randomized controlled trials; ROP: Retinopathy of prematurity; RR: Relative risk; WMD: Weighted mean difference

\section{Acknowledgements}

The authors would like to thank Professor Gabriel Altit of Universite de Montreal - CHU Sainte-Justine and Professor Gauthier Loron of Institut Alix de Champagne for advice and technical assistance.

\section{Funding}

This work was supported by Health and Family Planning Commission of Zhejiang Province of China (grant number 2014RCA021), Natural Science Foundation of Zhejiang Province (grant number LY15H040002), and the National Natural Science Foundation of China (grant number $81471480,81630037)$. The funding source play no role in study design, data collection, data analysis, data interpretation, writing of the report, or in the decision to submit the report for publication.

\section{Availability of data and materials}

All data generated or analysed during this study are included in this published article (and its supplementary information files).

\section{Authors' contributions}

Conception and design: LZD, ZQZ; Acquisition of data: ZQZ, YZ, XMH; Analysis and interpretation of data: LZD, ZQZ, YZ; Drafting the article: ZQZ; Revising the article critically for important intellectual content: LZD, ZQZ, YZ, $\mathrm{XMH}$; Final approval of the version to be published: LZD, ZQZ, YZ, XMH; Agreement to be accountable for all aspects of the work in ensuring that questions related to the accuracy or integrity of any part of the work are appropriately investigated and resolved: LZD, ZQZ, YZ, XMH. All of the authors read and approved the manuscript.
Ethics approval and consent to participate

As the paper did not involve any human or animal, the ethical approval was not required.

\section{Consent for publication}

Not applicable.

\section{Competing interests}

None of the investigators declare any real or perceived conflicts of interest pertaining to the subject of this manuscript.

\section{Publisher's Note}

Springer Nature remains neutral with regard to jurisdictional claims in published maps and institutional affiliations.

\section{Author details}

${ }^{1}$ Department of Neonatology, the Children's Hospital, Zhejiang University School of Medicine, No. 3333 Bingsheng Road, Hangzhou City, Zhejiang 310002, China. ${ }^{2}$ Department of Pediatrics, Hangzhou First People's Hospital, Nanjing Medical University, No. 261 Huansha Road, Hangzhou City, Zhejiang 310002, China.

Received: 18 June 2017 Accepted: 30 November 2017

Published online: 15 December 2017

\section{References}

1. Stoll BJ, Hansen NI, Bell EF, Shankaran S, Laptook AR, Walsh MC, et al. Trends in care practices, morbidity, and mortality of extremely preterm neonates, 1993-2012. JAMA. 2015:314:1039-51.

2. Walsh MC, Morris BH, Wrage LA, Vohr BR, Poole WK, Tyson JE, et al. Extremely low birthweight neonates with protracted ventilation: mortality and 18-month neurodevelopmental outcomes. J Pediatr. 2005;146:798-804.

3. Doyle LW, Faber B, Callanan C, Freezer N, Ford GW, Davis NM. Bronchopulmonary dysplasia in very low birth weight subjects and lung function in late adolescence. Pediatrics. 2006:118:108-13.

4. Short EJ, Kirchner HL, Asaad GR, Fulton SE, Lewis BA, Klein N, et al. Developmental sequelae in preterm infants having a diagnosis of bronchopulmonary dysplasia: analysis using a severity-based classification system. Arch Pediatr Adolesc Med. 2007;161:1082-7.

5. Kinsella JP, Greenough A, Abman SH. Bronchopulmonary dysplasia. Lancet. 2006:367:1421-31.

6. Jobe AH, Bancalari E. Bronchopulmonary dysplasia. Am J Respir Crit Care Med. 2001;163:1723-9.

7. Pierce MR, Bancalari E. The role of inflammation in the pathogenesis of bronchopulmonary dysplasia. Pediatr Pulmonol. 1995;19:371-8.

8. Onland W, Offringa M, Cools F, De Jaegere AP, Rademaker $\mathrm{K}$, Blom H, et al. Systemic hydrocortisone to prevent Bronchopulmonary dysplasia in preterm infants (the STOP-BPD study); a multicenter randomized placebo controlled trial. BMC Pediatr. 2011;11:102.

9. Halliday HL, Patterson CC, Halahakoon CW. European multicenter steroid study group. A multicenter, randomized open study of early corticosteroid treatment (OSECT) in preterm infants with respiratory illness: comparison of early and late treatment and of dexamethasone and inhaled budesonide. Pediatrics. 2001;107:232-40.

10. Doyle LW, Ehrenkranz RA, Halliday HL. Early ( $<8$ days) postnata corticosteroids for preventing chronic lung disease in preterm infants. Cochrane Database Syst Rev. 2014:5:CD001146.

11. Doyle LW, Ehrenkranz RA, Halliday HL. Late (> 7 days) postnatal corticosteroids for chronic lung disease in preterm infants. Cochrane Database Syst Rev. 2014;5:CD001145.

12. Gulliver T, Morton R, Eid N. Inhaled corticosteroids in children with asthma: pharmacologic determinants of safety and efficacy and other clinical considerations. Pediatr Drugs. 2007;9(3):185-94.

13. Mokra D, Mokry J, Drgova A, Petraskova M, Bulikova J, Calkovska A. Intratracheally administered corticosteroids improve lung function in meconium-instilled rabbits. J Physiol Pharmacol. 2007:5(Pt 1):389-98.

14. Onland $W$, Offringa $M$, van Kaam $A$. Late ( $\geq 7$ days) inhalation corticosteroids to reduce bronchopulmonary dysplasia in preterm infants. Cochrane Database Syst Rev. 2012;4:CD002311. 
15. Shah VS, Ohlsson A, Halliday HL, Dunn M. Early administration of inhaled corticosteroids for preventing chronic lung disease in very low birth weight preterm neonates. Cochrane Database Syst Rev. 2017;1:CD001969.

16. Bassler D, Plavka R, Shinwell ES, Hallman M, Jarreau PH, Carnielli V, et al. Early inhaled Budesonide for the prevention of Bronchopulmonary dysplasia. N Engl J Med. 2015;373:1497-506.

17. Shinwell ES, Portnov I, Meerpohl JJ, Karen T, Bassler D. Inhaled corticosteroids for Bronchopulmonary dysplasia: a meta-analysis. Pediatrics. 2016;138:e20162511.

18. Pokriefka E, Mehdizadeh B, Rabbani A. Inhaled flunisolide in bronchopulmonary dysplasia [abstract]. Pediatr Res. 1993;33:341A.

19. Dugas MA, Nguyen $D$, Frenette $L$, Lachance $C$, St-Onge $O$, Fougères $A$, et al. Fluticasone inhalation in moderate cases of bronchopulmonary dysplasia. Pediatrics. 2005;115:566-72

20. Yong WSC, Carney S, Pearse RG, Gibson AT. The effect of inhaled fluticasone propionate (FP) on premature babies at risk for developing chronic lung disease of prematurity [abstract]. Arch Dis Child. 1999;80:64

21. Shah SS, Ohlsson A, Halliday HL, Shah VS. Inhaled versus systemic corticosteroids for preventing chronic lung disease in ventilated very low birth weight preterm neonates. Cochrane Database Syst Rev. 2012;5:CD002058.

22. Shah SS, Ohlsson A, Halliday HL, Shah VS. Inhaled versus systemic corticosteroids for the treatment of chronic lung disease in ventilated very low birth weight preterm infants. Cochrane Database Syst Rev. 2012;5:CD002057

23. Schmidt B. No end to uncertainty about inhaled Glucocorticoids in preterm infants. N Engl J Med. 2015;373:1566-7.

24. Nakamura T, Yonemoto N, Nakayama M, Hirano S, Aotani H, Kusuda S, et al. Early inhaled steroid use in extremely low birthweight infants: a randomised controlled trial. Arch Dis Child Fetal Neonatal Ed. 2016;101:552-6.

25. Yeh TF, Chen CM, Wu SY, Husan Z, Li TC, Hsieh WS, et al. Intra-tracheal Administration of Budesonide/surfactant to prevent Bronchopulmonary dysplasia. Am J Respir Crit Care Med. 2016;193:86-95.

26. Moher D, Liberati A, Tetzlaff J, Altman DG, PRISMA Group. Preferred reporting items for systematic reviews and meta-analyses: the PRISMA statement. BMJ. 2009;339:b2535.

27. Zhiqun Z, Ying Z, Xianmei H, Lizhong D. Inhaled corticosteroids for prevention of bronchopulmonary dysplasia in premature infants: a systematic review and meta-analysis with trial sequential analysis. PROSPERO. 2016;2016:CRD42016054098.

28. Higgins JPT, Green S. Cochrane handbook for systematic reviews of interventions. 2011;Version 5.1.0. Cochrane Collaboration.

29. Wan X, Wang W, Liu J, Tong T. Estimating the sample mean and standard deviation from the sample size, median, range and/or interquartile range. BMC Med Res Methodol. 2014; https://doi.org/10.1186/1471-2288-14-135.

30. Higgins JP, Thompson SG. Quantifying heterogeneity in a meta-analysis. Stat Med. 2002;21:1539-58.

31. Thorlund K, Devereaux PJ, Wetterslev J, Guyatt G, loannidis JP, Thabane L, et al. Can trial sequential monitoring boundaries reduce spurious inferences from meta-analyses? Int J Epidemiol. 2009;38:276-86.

32. Thorlund K, Engstrøm J, Wetterslev J, Brok J, Imberger G, Gluud C. User manual for trial sequential analysis (TSA). Copenhagen trial unit. Centre for clinical intervention research, 2011.

33. Wetterslev J, Thorlund K, Brok J, Gluud C. Trial sequential analysis may establish when firm evidence is reached in cumulative meta-analysis. J Clin Epidemiol. 2008;61:64-75.

34. Wetterslev J, Thorlund K, Brok J, Gluud C. Estimating required information size by quantifying diversity in random-effects model meta-analyses. BMC Med Res Methodol. 2009; https://doi.org/10.1186/1471-2288-9-86.

35. Arnon S, Grigg J, Silverman M. Effectiveness of budesonide aerosol in ventilator-dependent preterm babies: a preliminary report. Pediatr Pulmonol. 1996:21:231-5.

36. Cao Y, Shi B. Clinical study of combined aerosol inhalation of budesonide and pulmonary surfactant to prevent bronchopulmonary dysplasia. Taishan: Taishan Medical University; 2016.

37. Cole CH, Colton T, Shah BL, Abbasi S, Mackinnon BL, Demissie S, Frantz ID 3rd. Early inhaled glucocorticoid therapy to prevent bronchopulmonary dysplasia. N Engl J Med. 1999;340:1005-10.

38. Denjean A, Paris-Llado J, Zupan V, Debillon T, Kieffer F, Magny JF, et al. Inhaled salbutamol and beclomethasone for preventing bronchopulmonary dysplasia: a randomised double-blind study. Eur J Pediatr. 1998;157:926-31.
39. Fok TF, Lam K, Dolovich M, Ng PC, Wong W, Cheung KL, et al. Randomised controlled study of early use of inhaled corticosteroid in preterm infants with respiratory distress syndrome. Arch Dis Child Fetal Neonatal Ed. 1999;80:203-8.

40. Giep T, Raibble P, Zuerlein T, Schwartz ID. Trial of beclomethasone dipropionate by metered-dose inhaler in ventilator-dependent neonates less than 1500 grams. Am J Perinatol. 1996;13:5-9.

41. Jangaard KA, Stinson DA, Allen AC, Vincer MJ. Early prophylactic inhaled beclomethasone in infants less than $1250 \mathrm{~g}$ for the prevention of chronic lung disease. Paediatr Child Health. 2002;7:13-9.

42. Jónsson B, Eriksson M, Söder O, Broberger U, Lagercrantz H. Budesonide delivered by dosimetric jet nebulization to preterm very low birthweight infants at high risk for development of chronic lung disease. Acta Paediatr. 2000;89:1449-55.

43. Ke H, Li ZK, Yu XP, Guo JZ. Efficacy of different preparations of budesonide combined with pulmonary surfactant in the treatment of neonatal respiratory distress syndrome: a comparative analysis. Chinese Journal of Contemporary Pediatrics. 2016;18:400-4.

44. LaForce WR, Brudno DS. Controlled trial of beclomethasone dipropionate by nebulization in oxygen- and ventilator-dependent infants. J Pediatr. 1993;122:285-8.

45. Merz U, Kusenbach G, Häusler M, Peschgens T, Hörnchen H. Inhaled budesonide in ventilator-dependent preterm infants: a randomized, doubleblind pilot study. Biol Neonate. 1999;75:46-53.

46. Pappagallo M, Abbasi S, Bhutani VK. Respiratory and systemic effects of inhaled dexamethasone on ventilator dependant preterm infants at risk for bronchopulmonary dysplasia. Indian J Pediatr. 1998;65:273-82.

47. Townsend SF, Hale KA, Thilo EH. Early treatment with inhaled steroids does not improve outcome in extremely premature infants with respiratory. Distress [abstract]. Pediatr Res. 1998;43:300A.

48. Wen M, Wang R, Chen D. Clinical observation of budesonide suspension preventing premature infants with bronchopulmonary dysplasia. World Latest Medicne Information. 2016;16:85-6.

49. Yeh TF, Lin HC, Chang CH, Wu TS, Su BH, Li TC, et al. Early airway instillation of budesonide using surfactant as a vehicle to prevent chronic lung disease in preterm infants: a pilot study. Pediatrics. 2008;121:1310-8.

50. Kuo HT, Lin HC, Tsai CH, Chouc IC, Yeh TF. A follow-up study of preterm infants given budesonide using surfactant as a vehicle to prevent chronic lung disease in preterm infants. J Pediatr. 2010;156:537-41.

51. Zimmerman JJ, Gabbert D, Shivpuri C, Kayata S, Miller J, Ciesielski W. Meterdosed, inhaled beclomethasone initiated at birth to prevent bronchopulmonary dysplasia. Pediatr Crit Care Med. 2000;1:140-5.

52. Dimitriou G, Greenough A, Giffin FJ, Kavadia V. Inhaled versus systemic steroids in chronic oxygen dependency of preterm infants. Eur J Pediatr. 1997;156:51-5.

53. Groneck P, Goetze-Speer B, Speer CP. Effects of inhaled beclomethasone compared to systemic dexamethasone on lung inflammation in preterm infants at risk of chronic lung disease. Pediatr Pulmonol. 1999;27:383-7.

54. Halliday HL, Patterson CC, Halahakoon CW, European Multicenter Steroid Study Group. European Multicenter Steroid Study Group. A multicenter, randomized open study of early corticosteroid treatment (OSECT) in preterm infants with respiratory illness: comparison of early and late treatment and of dexamethasone and inhaled budesonide. Pediatrics. 2001;107:232-40.

55. Wilson TT, Waters L, Patterson CC, McCusker CG, Rooney NM, Marlow $\mathrm{N}$, et al. Neurodevelopmental and respiratory follow-up results at 7 years for children from the United Kingdom and Ireland enrolled in a randomized trial of early and late postnatal corticosteroid treatment, systemic and inhaled (the open study of early corticosteroid treatment). Pediatrics. 2006;117:2196-205.

56. Rozycki HJ, Byron PR, Elliott GR, Carroll T, Gutcher GR. Randomized controlled trial of three different doses of aerosol beclomethasone versus systemic dexamethasone to promote extubation in ventilated premature infants. Pediatr Pulmonol. 2003;35:375-83.

57. Suchomski SJ, Cummings JJ. A randomized trial of inhaled versus intravenous steroids in ventilator-dependent preterm infants. J Perinatol. 2002;22:196-203.

58. Beresford MW, Primhak R, Subhedar NV, Shaw NJ. Randomised double blind placebo controlled trial of inhaled fluticasone propionate in infants with chronic lung disease. Arch Dis Child Fetal Neonatal Ed. 2002;87:62-3.

59. Kugelman A, Peniakov M, Zangen S, Shiff Y, Riskin A, lofe A, et al. Inhaled hydrofluoalkane-beclomethasone dipropionate in bronchopulmonary dysplasia. A double-blind, randomized, controlled pilot study. J Perinatol. 2017;37:197-202. 
60. Parikh NA, Locke RG, Chidekel A, Leef KH, Emberger J, Paul DA, et al. Effect of inhaled corticosteroids on markers of pulmonary inflammation and lung maturation in preterm infants with evolving chronic lung disease. J Am Osteopath Assoc. 2004:104:114-20.

61. Nicholl RM, Greenough A, King M, Cheeseman P, Gamsu HR. Growth effects of systemic versus inhaled steroids in chronic lung disease. Arch Dis Child Fetal Neonatal Ed. 2002;87:59-61.

62. Cole CH, Shah B, Abbasi S, Demissie S, Mackinnon B, Colton T, Frantz ID 3rd. Adrenal function in premature infants during inhaled beclomethasone therapy. J Pediatr. 1999;135:65-70.

63. Ng PC, Fok TF, Wong GW, Lam CW, Lee CH, Wong MY, et al. Pituitaryadrenal suppression in preterm, very low birth weight infants after inhaled fluticasone propionate treatment. J Clin Endocrinol Metab. 1998;83:2390-3.

64. Sweet D, Bevilacqua G, Carnielli V, Greisen G, Plavka R, Saugstad OD, et al. European Association of Perinatal Medicine. European consensus guidelines on the management of neonatal respiratory distress syndrome. J Perinat Med. 2007;35:175-86.

65. Sweet DG, Carnielli V, Greisen G, Hallman M, Ozek E, Plavka R, et al. European consensus guidelines on the management of neonatal respiratory distress syndrome in preterm infants - 2010 update. Neonatology. 2010;97:402-17.

66. Sweet DG, Carnielli V, Greisen G, Hallman M, Ozek E, Plavka R, et al. European consensus guidelines on the management of neonatal respiratory distress syndrome in preterm infants-2013 update. Neonatology. 2013;103:353-68.

67. Wiedmann TS, Bhatia R, Wattenberg LW. W. Drug solubilization in lung surfactant. J Control Release. 2000;65:43-7.

68. Sweet DG, Carnielli V, Greisen G, Hallman M, Ozek E, Plavka R, et al. European consensus guidelines on the Management of Respiratory Distress Syndrome -2016 update. Neonatology. 2016;111:107-25.

69. Guyatt GH, Oxman AD, Vist GE, Kunz R, Falck-Ytter Y, Alonso-Coello P, et al. GRADE: an emerging consensus on rating quality of evidence and strength of recommendations. BMJ. 2008;336:924-6.

70. Hines D, Modi N, Lee SK, Isayama T, Sjors G, Gagliardi L, et al. Scoping review shows wide variation in the definitions of bronchopulmonary dysplasia in preterm infants and calls for a consensus. Acta Paediatr. 2017;106:366-74.

\section{Submit your next manuscript to BioMed Central and we will help you at every step:}

- We accept pre-submission inquiries

- Our selector tool helps you to find the most relevant journal

- We provide round the clock customer support

- Convenient online submission

- Thorough peer review

- Inclusion in PubMed and all major indexing services

- Maximum visibility for your research

Submit your manuscript at www.biomedcentral.com/submit 